\title{
Unified wave field retrieval and imaging method for inhomogeneous non-reciprocal media
}

\author{
Kees Wapenaar and Christian Reinicke \\ Department of Geoscience and Engineering, \\ Delft University of Technology, \\ Stevinweg 1, \\ 2628 CN Delft, \\ The Netherlands
}

\begin{abstract}
Acoustic imaging methods often ignore multiple scattering. This leads to false images in cases where multiple scattering is strong. Marchenko imaging has recently been introduced as a datadriven way to deal with internal multiple scattering.

Given the increasing interest in non-reciprocal materials, both for acoustic and electromagnetic applications, we propose to modify the Marchenko method for imaging such materials. We formulate a unified wave equation for non-reciprocal materials, exploiting the similarity between acoustic and electromagnetic wave phenomena. This unified wave equation forms the basis for deriving reciprocity theorems that interrelate wave fields in a non-reciprocal medium and its complementary version. Next, we reformulate these theorems for downgoing and upgoing wave fields. From these decomposed reciprocity theorems we derive representations of the Green's function inside the nonreciprocal medium, in terms of the reflection response at the surface and focusing functions inside the medium and its complementary version. These representations form the basis for deriving a modified version of the Marchenko method to retrieve the wave field inside a non-reciprocal medium and to form an image, free from artefacts related to multiple scattering. We illustrate the proposed method at the hand of the numerically modeled reflection response of a horizontally layered medium.
\end{abstract}




\section{INTRODUCTION}

Acoustic imaging methods are traditionally based on the single-scattering assumption [115]. Multiply scattered waves are not properly handled by these methods and may lead to false images overlaying the desired primary image. Several approaches have been developed that account for multiple scattering. For the sake of the discussion it is important to distinguish between different classes of multiply scattered waves. Waves that have scattered at least once at the surface of the medium are called surface-related multiples. This type of multiple scattering is particularly severe in exploration geophysics. However, because the scattering boundary is known, this class of multiples is relatively easily dealt with. Successful methods have been developed to suppress surface-related multiples prior to imaging [16-21]. Waves that scatter several times inside the medium before being recorded at the surface are called internal multiples. Internal multiple scattering may occur at heterogeneities at many scales. We may distinguish between deterministic scattering at well-separated scatterers, giving rise to long period multiples, and diffuse scattering in stochastic media. Of course this distinction is not always sharp. In this paper we only consider the first type of internal multiple scattering, which typically occurs in layered media (which, in general, may have curved interfaces and varying parameters in the layers). Several imaging approaches that account for deterministic internal multiples are currently under development, such as the inverse scattering series approach [22 24], full wave field migration [25, 26], and Marchenko imaging. The latter approach builds on a 1D autofocusing procedure [27 29], which has been generalised for 2D and 3D inhomogeneous media [30-41]. This methodology retrieves the wave fields inside a medium, including all internal multiples, in a data-driven way. Such wave fields could be used, for example, to monitor changes of the material over time. Moreover, in a next step these wave fields can be used to form an image of the material, in which artefacts due to the internal multiples are suppressed. Promising results have been obtained with geophysical [42 46] and ultrasonic data [46, 47].

To date, the application of the Marchenko method has been restricted to reciprocal media. With the increasing interest in non-reciprocal materials, both in electromagnetics [48 50] and in acoustics and elastodynamics [51 57], it is opportune to modify the Marchenko method for non-reciprocal media. We start with a brief review of the wave equation for non-reciprocal media. By restricting this to scalar waves in a $2 \mathrm{D}$ plane, it is possible to capture different wave phenomena by a unified wave equation. Next, we formulate reciprocity theorems for waves in a non-reciprocal medium and its complementary version (the complementary medium will be defined later). From these reciprocity theorems we derive Green's function representations, which form the basis for the Marchenko method in non-reciprocal media. We illustrate the new method with a numerical example, showing that it has the potential 
to accurately retrieve the wave fields inside a non-reciprocal medium and to image this medium, without false images related to multiply scattered waves.

\section{UNIFIED WAVE EQUATION FOR NON-RECIPROCAL MEDIA}

Consider the following unified equations in the low-frequency limit for 2D wave propagation in the $\left(x_{1}, x_{3}\right)$-plane in inhomogeneous, lossless, anisotropic, non-reciprocal media

$$
\begin{aligned}
& \alpha \partial_{t} P+\left(\partial_{r}+\gamma_{r} \partial_{t}\right) Q_{r}=B \\
& \left(\partial_{r}+\gamma_{r} \partial_{t}\right) P+\beta_{r s} \partial_{t} Q_{s}=C_{r} .
\end{aligned}
$$

These equations hold for transverse-electric (TE), transverse-magnetic (TM), horizontallypolarised shear $(\mathrm{SH})$ and acoustic $(\mathrm{AC})$ waves. They are formulated in the space-time $(\mathbf{x}, t)$ domain, with $\mathbf{x}=\left(x_{1}, x_{3}\right)$. Operator $\partial_{r}$ stands for differentiation in the $x_{r}$ direction. Lowercase subscripts $r$ and $s$ take the values 1 and 3 only; Einstein's summation convention applies for repeated subscripts. Operator $\partial_{t}$ stands for temporal differentiation. The wave field quantities $\left(P=P(\mathbf{x}, t)\right.$ and $\left.Q_{r}=Q_{r}(\mathbf{x}, t)\right)$ and source quantities $(B=B(\mathbf{x}, t)$ and $\left.C_{r}=C_{r}(\mathbf{x}, t)\right)$ are macroscopic quantities. These are often denoted as $\langle P\rangle$ etc. [48], but for notational convenience we will not use the brackets. The medium parameters $(\alpha=\alpha(\mathbf{x})$, $\beta_{r s}=\beta_{r s}(\mathbf{x})$ and $\left.\gamma_{r}=\gamma_{r}(\mathbf{x})\right)$ are effective parameters. In general they are anisotropic at macro scale (with $\beta_{r s}=\beta_{s r}$ ), even when they are isotropic at micro scale. Wave field quantities, source quantities and medium parameters are specified for the different wave phenomena in Table 1. For TE and TM waves, the macroscopic wave field quantities are $E$ (electric field strength) and $H$ (magnetic field strength), the macroscopic source functions are $J^{\mathrm{e}}$ (external electric current density) and $J^{\mathrm{m}}$ (external magnetic current density), and the effective medium parameters are $\varepsilon^{o}$ (permittivity), $\mu$ (permeability) and $\xi$ (coupling parameter). For $\mathrm{SH}$ and $\mathrm{AC}$ waves, the macroscopic wave field quantities are $v$ (particle velocity), $\tau$ (stress) and $p$ (acoustic pressure), the macroscopic source functions are $F$ (external force density), $h$ (external deformation-rate density) and $q$ (volume injection-rate density), and the effective medium parameters are $\rho^{o}$ (mass density), $s$ (compliance), $\kappa$ (compressibility) and $\xi$ (coupling parameter). For further details we refer to Appendix A. 
Table 1: Quantities in unified equations (1) and (2).

\begin{tabular}{|c|c|c|c|c|c|c|c|c|c|c|c|c|}
\hline \hline & $P$ & $Q_{1}$ & $Q_{3}$ & $\alpha$ & $\beta_{11}$ & $\beta_{31}$ & $\beta_{33}$ & $\gamma_{1}$ & $\gamma_{3}$ & $B$ & $C_{1}$ & $C_{3}$ \\
\hline $\mathrm{TE}$ & $E_{2}$ & $H_{3}$ & $-H_{1}$ & $\varepsilon_{22}^{o}$ & $\mu_{33}$ & $-\mu_{31}$ & $\mu_{11}$ & $\xi_{23}$ & $-\xi_{21}$ & $-J_{2}^{\mathrm{e}}$ & $-J_{3}^{\mathrm{m}}$ & $J_{1}^{\mathrm{m}}$ \\
\hline $\mathrm{TM}$ & $H_{2}$ & $-E_{3}$ & $E_{1}$ & $\mu_{22}$ & $\varepsilon_{33}^{o}$ & $-\varepsilon_{31}^{o}$ & $\varepsilon_{11}^{o}$ & $-\xi_{32}$ & $\xi_{12}$ & $-J_{2}^{\mathrm{m}}$ & $J_{3}^{\mathrm{e}}$ & $-J_{1}^{\mathrm{e}}$ \\
\hline $\mathrm{SH}$ & $v_{2}$ & $-\tau_{21}$ & $-\tau_{23}$ & $\rho_{22}^{o}$ & $4 s_{1221}$ & $4 s_{1223}$ & $4 s_{3223}$ & $2 \xi_{221}$ & $2 \xi_{223}$ & $F_{2}$ & $2 h_{21}$ & $2 h_{23}$ \\
\hline $\mathrm{AC}$ & $p$ & $v_{1}$ & $v_{3}$ & $\kappa$ & $\rho_{11}^{o}$ & $\rho_{31}^{o}$ & $\rho_{33}^{o}$ & $\xi_{1}$ & $\xi_{3}$ & $q$ & $F_{1}$ & $F_{3}$ \\
\hline \hline
\end{tabular}

By eliminating $Q_{r}$ from equations (1) and (2) we obtain a scalar wave equation for field quantity $P$, according to

$$
\left(\partial_{r}+\gamma_{r} \partial_{t}\right) \vartheta_{r s}\left(\partial_{s}+\gamma_{s} \partial_{t}\right) P-\alpha \partial_{t}^{2} P=\left(\partial_{r}+\gamma_{r} \partial_{t}\right) \vartheta_{r s} C_{s}-\partial_{t} B
$$

see Appendix A for the derivation. Here $\vartheta_{r s}$ is the inverse of $\beta_{r s}$. Compare equation (3) with the common scalar wave equation for waves in isotropic reciprocal media

$$
\partial_{r} \frac{1}{\beta} \partial_{r} P-\alpha \partial_{t}^{2} P=\partial_{r} \frac{1}{\beta} C_{r}-\partial_{t} B
$$

In equation (3), $\partial_{r}+\gamma_{r} \partial_{t}$ replaces $\partial_{r}$, with $\gamma_{r}$ being responsible for the non-reciprocal behaviour. Moreover, $\vartheta_{r s}$ replaces $1 / \beta$, thus accounting for anisotropy of the effective nonreciprocal medium.

To illustrate the physical meaning of the parameter $\gamma_{r}$, we consider the $1 \mathrm{D}$ version of equation (3) for a homogeneous, isotropic, source-free medium, i.e.

$$
\left(\partial_{1}+\gamma \partial_{t}\right)\left(\partial_{1}+\gamma \partial_{t}\right) P-\alpha \beta \partial_{t}^{2} P=0
$$

Its solution reads

$$
P^{ \pm}\left(x_{1}, t\right)=S\left(t \mp \frac{x_{1}}{c}(1 \pm \gamma c)\right)
$$

with $S(t)$ being an arbitrary time-dependent function and $c=(\alpha \beta)^{-1 / 2}$ the propagation velocity of the corresponding reciprocal medium. Note that $P^{+}\left(x_{1}, t\right)$ propagates in the positive $x_{1}$-direction with slowness $(1+\gamma c) / c$, whereas $P^{-}\left(x_{1}, t\right)$ propagates in the negative $x_{1}$-direction with slowness $(1-\gamma c) / c$. Hence, $\gamma$ determines the asymmetry of the slownesses in opposite directions. Throughout this paper we assume that $\left|\gamma_{r}\right|$ is smaller than the lowest inverse propagation velocity of the corresponding reciprocal anisotropic medium. 


\section{RECIPROCITY THEOREMS FOR A NON-RECIPROCAL MEDIUM AND ITS COMPLEMENTARY VERSION}

We derive reciprocity theorems in the space-frequency $(\mathbf{x}, \omega)$-domain for wave fields in a non-reciprocal medium and its complementary version. To this end, we define the temporal Fourier transform of a space- and time-dependent function $P(\mathbf{x}, t)$ as

$$
P(\mathbf{x}, \omega)=\int_{-\infty}^{\infty} P(\mathbf{x}, t) \exp (i \omega t) \mathrm{d} t
$$

where $\omega$ is the angular frequency and $i$ the imaginary unit. For notational convenience we use the same symbol for quantities in the time domain and in the frequency domain. We use equation (7) to transform equations (1) and (2) to the space-frequency domain. The temporal differential operators $\partial_{t}$ are thus replaced by $-i \omega$, hence

$$
\begin{aligned}
& -i \omega \alpha P+\left(\partial_{r}-i \omega \gamma_{r}\right) Q_{r}=B \\
& \left(\partial_{r}-i \omega \gamma_{r}\right) P-i \omega \beta_{r s} Q_{s}=C_{r},
\end{aligned}
$$

with $P=P(\mathbf{x}, \omega), Q_{r}=Q_{r}(\mathbf{x}, \omega), B=B(\mathbf{x}, \omega)$ and $C_{r}=C_{r}(\mathbf{x}, \omega)$. A reciprocity theorem formulates a mathematical relation between two independent states [58 60]. We indicate the wave fields, sources and medium parameters in the two states by subscripts $A$ and $B$. Consider the quantity

$$
\partial_{r}\left(P_{A} Q_{r, B}-Q_{r, A} P_{B}\right)
$$

Applying the product rule for differentiation, using equations (8) and (9) for states $A$ and $B$, using $\beta_{s r}=\beta_{r s}$ [56, 61, 62], integrating the result over domain $\mathbb{D}$ enclosed by boundary $\partial \mathbb{D}$ with outward pointing normal vector $\mathbf{n}=\left(n_{1}, n_{3}\right)$ and applying the theorem of Gauss, we obtain

$$
\begin{aligned}
& \oint_{\partial \mathbb{D}}\left(P_{A} Q_{r, B}-Q_{r, A} P_{B}\right) n_{r} \mathrm{~d} \mathbf{x}= \\
& i \omega \int_{\mathbb{D}}\left(\left(\alpha_{B}-\alpha_{A}\right) P_{A} P_{B}-\left(\beta_{r s, B}-\beta_{r s, A}\right) Q_{r, A} Q_{s, B}\right) \mathrm{d} \mathbf{x} \\
& +i \omega \int_{\mathbb{D}}\left(\gamma_{r, B}+\gamma_{r, A}\right)\left(P_{A} Q_{r, B}-Q_{r, A} P_{B}\right) \mathrm{d} \mathbf{x} \\
& +\int_{\mathbb{D}}\left(C_{r, A} Q_{r, B}-Q_{r, A} C_{r, B}+P_{A} B_{B}-B_{A} P_{B}\right) \mathrm{d} \mathbf{x} .
\end{aligned}
$$

This is the general reciprocity theorem of the convolution type. When the medium parameters $\alpha, \beta_{r s}$ and $\gamma_{r}$ are identical in both states, then the first integral on the right-hand 
side vanishes, but the second integral, containing $\gamma_{r}$, does not vanish. When we choose $\gamma_{r, A}=-\gamma_{r, B}=-\gamma_{r}$, then the second integral also vanishes. For this situation we call state $B$, with parameters $\alpha, \beta_{r s}$ and $\gamma_{r}$, the actual state, and state $A$, with parameters $\alpha, \beta_{r s}$ and $-\gamma_{r}$, the complementary state [61, 63] (also known as the Lorentz-adjoint state [64]). We indicate the complementary state by a superscript (c). Hence

$$
\begin{aligned}
& \oint_{\partial \mathbb{D}}\left(P_{A}^{(\mathrm{c})} Q_{r, B}-Q_{r, A}^{(\mathrm{c})} P_{B}\right) n_{r} \mathrm{~d} \mathbf{x}= \\
& \oint_{\mathbb{D}}\left(C_{r, A}^{(\mathrm{c})} Q_{r, B}-Q_{r, A}^{(\mathrm{c})} C_{r, B}+P_{A}^{(\mathrm{c})} B_{B}-B_{A}^{(\mathrm{c})} P_{B}\right) \mathrm{d} \mathbf{x} .
\end{aligned}
$$

This reciprocity theorem will play a role in the derivation of Green's function representations for the Marchenko method for non-reciprocal media (section IV]). Here we use it to derive a relation between Green's functions in states $A$ and $B$. For the complementary state $A$ we choose a unit monopole point source at $\mathbf{x}_{S}$ in $\mathbb{D}$, hence $B_{A}^{(\mathrm{c})}(\mathbf{x}, \omega)=\delta\left(\mathbf{x}-\mathbf{x}_{S}\right)$, where $\delta(\mathbf{x})$ is the Dirac delta function. The response to this point source is the Green's function in state $A$, hence $P_{A}^{(\mathrm{c})}(\mathbf{x}, \omega)=G^{(\mathrm{c})}\left(\mathbf{x}, \mathbf{x}_{S}, \omega\right)$. Similarly, for state $B$ we choose a unit monopole point source at $\mathbf{x}_{R}$ in $\mathbb{D}$, hence $B_{B}(\mathbf{x}, \omega)=\delta\left(\mathbf{x}-\mathbf{x}_{R}\right)$ and $P_{B}(\mathbf{x}, \omega)=G\left(\mathbf{x}, \mathbf{x}_{R}, \omega\right)$. We substitute these expressions into equation 12 and set the other source quantities, $C_{r, A}^{(\mathrm{c})}$ and $C_{r, B}$, to zero. Further, we assume that Neumann or Dirichlet boundary conditions apply at $\partial \mathbb{D}$, or that the medium at and outside $\partial \mathbb{D}$ is homogeneous and reciprocal. In each of these cases the boundary integral vanishes. We thus obtain [51, 65]

$$
G^{(\mathrm{c})}\left(\mathbf{x}_{R}, \mathbf{x}_{S}, \omega\right)=G\left(\mathbf{x}_{S}, \mathbf{x}_{R}, \omega\right)
$$

The left-hand side is the response to a source at $\mathbf{x}_{S}$ in the complementary medium (with parameter $-\gamma_{r}$ ), observed by a receiver at $\mathbf{x}_{R}$. The right-hand side is the response to a source at $\mathbf{x}_{R}$ in the actual medium (with parameter $\gamma_{r}$ ), observed by a receiver at $\mathbf{x}_{S}$. Note the analogy with the flow-reversal theorem for waves in flowing media [66 68].

Next, we consider the quantity

$$
\partial_{r}\left(P_{A}^{*} Q_{r, B}+Q_{r, A}^{*} P_{B}\right)
$$




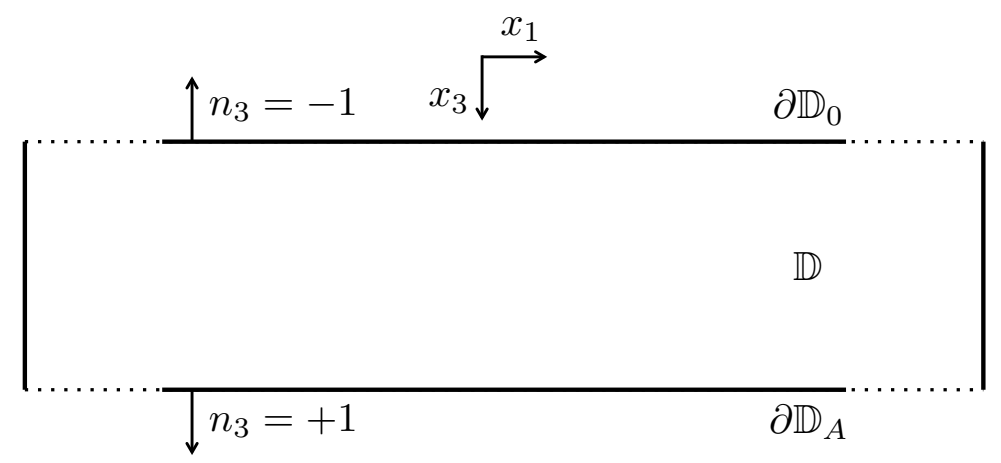

FIG. 1: Modified configuration for the reciprocity theorems.

Superscript $*$ denotes complex conjugation. Following the same steps as before, we obtain

$$
\begin{aligned}
& \oint_{\partial \mathbb{D}}\left(P_{A}^{*} Q_{r, B}+Q_{r, A}^{*} P_{B}\right) n_{r} \mathrm{~d} \mathbf{x}= \\
& i \omega \int_{\mathbb{D}}\left(\left(\alpha_{B}-\alpha_{A}\right) P_{A}^{*} P_{B}+\left(\beta_{r s, B}-\beta_{r s, A}\right) Q_{r, A}^{*} Q_{s, B}\right) \mathrm{d} \mathbf{x} \\
& +i \omega \int_{\mathbb{D}}\left(\gamma_{r, B}-\gamma_{r, A}\right)\left(P_{A}^{*} Q_{r, B}+Q_{r, A}^{*} P_{B}\right) \mathrm{d} \mathbf{x} \\
& +\int_{\mathbb{D}}\left(C_{r, A}^{*} Q_{r, B}+Q_{r, A}^{*} C_{r, B}+P_{A}^{*} B_{B}+B_{A}^{*} P_{B}\right) \mathrm{d} \mathbf{x} .
\end{aligned}
$$

This is the general reciprocity theorem of the correlation type. When the medium parameters $\alpha, \beta_{r s}$ and $\gamma_{r}$ are identical in both states, then the first and second integral on the right-hand side vanish. Hence

$$
\begin{aligned}
& \oint_{\partial \mathbb{D}}\left(P_{A}^{*} Q_{r, B}+Q_{r, A}^{*} P_{B}\right) n_{r} \mathrm{~d} \mathbf{x}= \\
& \oint_{\mathbb{D}}\left(C_{r, A}^{*} Q_{r, B}+Q_{r, A}^{*} C_{r, B}+P_{A}^{*} B_{B}+B_{A}^{*} P_{B}\right) \mathrm{d} \mathbf{x} .
\end{aligned}
$$

Also this reciprocity theorem will play a role in the derivation of Green's function representations for the Marchenko method for non-reciprocal media.

\section{GREEN'S FUNCTION REPRESENTATIONS FOR THE MARCHENKO METHOD}

We use the reciprocity theorems of the convolution and correlation type (equations (12) and (16p) to derive Green's function representations for the Marchenko method for nonreciprocal media. The derivation is similar to that for reciprocal media [31]; here we em- 
phasise the differences. We consider a spatial domain $\mathbb{D}$, enclosed by two infinite horizontal boundaries $\partial \mathbb{D}_{0}$ and $\partial \mathbb{D}_{A}$ (with $\partial \mathbb{D}_{A}$ below $\partial \mathbb{D}_{0}$ ), and two finite vertical side boundaries (at $\left.x_{1} \rightarrow \pm \infty\right)$, see Figure 1 . The positive $x_{3}$-axis points downward. The normal vectors at $\partial \mathbb{D}_{0}$ and $\partial \mathbb{D}_{A}$ are $\mathbf{n}=(0,-1)$ and $\mathbf{n}=(0,1)$, respectively. The boundary integrals in equations (12) and (16) along the vertical side boundaries vanish [69. Assuming there are no sources in $\mathbb{D}$ in both states, the reciprocity theorems thus simplify to

$$
\int_{\partial \mathbb{D}_{0}}\left(P_{A}^{(\mathrm{c})} Q_{3, B}-Q_{3, A}^{(\mathrm{c})} P_{B}\right) \mathrm{d} \mathbf{x}=\int_{\partial \mathbb{D}_{A}}\left(P_{A}^{(\mathrm{c})} Q_{3, B}-Q_{3, A}^{(\mathrm{c})} P_{B}\right) \mathrm{d} \mathbf{x}
$$

and

$$
\int_{\partial \mathbb{D}_{0}}\left(P_{A}^{*} Q_{3, B}+Q_{3, A}^{*} P_{B}\right) \mathrm{d} \mathbf{x}=\int_{\partial \mathbb{D}_{A}}\left(P_{A}^{*} Q_{3, B}+Q_{3, A}^{*} P_{B}\right) \mathrm{d} \mathbf{x} .
$$

For the derivation of the representations for the Marchenko method it is convenient to decompose the wave field quantities in these theorems into downgoing and upgoing fields in both states. Consider the following relations

$$
\mathbf{q}=\mathcal{L} \mathbf{p}, \quad \mathbf{p}=\mathcal{L}^{-1} \mathbf{q}
$$

with wave vectors $\mathbf{q}=\mathbf{q}(\mathbf{x}, \omega)$ and $\mathbf{p}=\mathbf{p}(\mathbf{x}, \omega)$ defined as

$$
\mathbf{q}=\left(\begin{array}{c}
P \\
Q_{3}
\end{array}\right), \quad \mathbf{p}=\left(\begin{array}{c}
U^{+} \\
U^{-}
\end{array}\right) .
$$

Here $U^{+}=U^{+}(\mathbf{x}, \omega)$ and $U^{-}=U^{-}(\mathbf{x}, \omega)$ are downgoing and upgoing flux-normalized wave fields, respectively. Operator $\mathcal{L}=\mathcal{L}(\mathbf{x}, \omega)$ in equation (19) is a pseudo-differential operator that composes the total wave field from its downgoing and upgoing constituents [69 77]. Its inverse decomposes the total wave field into downgoing and upgoing fields. For inhomogeneous isotropic reciprocal media, the theory for this operator is well developed. For anisotropic non-reciprocal media, we restrict the application of this operator to the laterally invariant situation. In Appendix $\mathrm{B}$ we use equations $(19)$ and $(20)$ at boundaries $\partial \mathbb{D}_{0}$ and $\partial \mathbb{D}_{A}$ to recast reciprocity theorems (17) and $(18)$ as follows

$$
\int_{\partial \mathbb{D}_{0}}\left(U_{A}^{+(\mathrm{c})} U_{B}^{-}-U_{A}^{-(\mathrm{c})} U_{B}^{+}\right) \mathrm{d} \mathbf{x}=\int_{\partial \mathbb{D}_{A}}\left(U_{A}^{+(\mathrm{c})} U_{B}^{-}-U_{A}^{-(\mathrm{c})} U_{B}^{+}\right) \mathrm{d} \mathbf{x}
$$


and

$$
\int_{\partial \mathbb{D}_{0}}\left(U_{A}^{+*} U_{B}^{+}-U_{A}^{-*} U_{B}^{-}\right) \mathrm{d} \mathbf{x}=\int_{\partial \mathbb{D}_{A}}\left(U_{A}^{+*} U_{B}^{+}-U_{A}^{-*} U_{B}^{-}\right) \mathrm{d} \mathbf{x} .
$$

Equation (21) is exact, whereas in equation (22) evanescent waves are neglected at boundaries $\partial \mathbb{D}_{0}$ and $\partial \mathbb{D}_{A}$. Note that the assumption of lateral invariance only applies to boundaries $\partial \mathbb{D}_{0}$ and $\partial \mathbb{D}_{A}$; the remainder of the medium (in- and outside $\mathbb{D}$ ) may be arbitrary inhomogeneous.

In the following we define $\partial \mathbb{D}_{0}\left(\right.$ at $\left.x_{3}=x_{3,0}\right)$ as the upper boundary of an inhomogeneous, anisotropic, non-reciprocal, lossless medium. Furthermore, we define $\partial \mathbb{D}_{A}$ (at $x_{3}=x_{3, A}$, with $\left.x_{3, A}>x_{3,0}\right)$ as an arbitrary boundary inside the medium. We assume that the medium above $\partial \mathbb{D}_{0}$ is homogeneous. For state $B$ we consider a unit source for downgoing waves at $\mathbf{x}_{S}=\left(x_{1, S}, x_{3, S}\right)$, just above $\partial \mathbb{D}_{0}$ (hence, $x_{3, S}=x_{3,0}-\epsilon$, with $\epsilon \rightarrow 0$ ). The response to this unit source at any observation point $\mathbf{x}$ is given by $U_{B}^{ \pm}(\mathbf{x}, \omega)=G^{ \pm}\left(\mathbf{x}, \mathbf{x}_{S}, \omega\right)$, where $G^{+}$and $G^{-}$denote the downgoing and upgoing components of the Green's function. For $\mathbf{x}$ at $\partial \mathbb{D}_{0}$, i.e., just below the source, we have $U_{B}^{+}(\mathbf{x}, \omega)=G^{+}\left(\mathbf{x}, \mathbf{x}_{S}, \omega\right)=\delta\left(x_{1}-x_{1, S}\right)$ and $U_{B}^{-}(\mathbf{x}, \omega)=G^{-}\left(\mathbf{x}, \mathbf{x}_{S}, \omega\right)=R\left(\mathbf{x}, \mathbf{x}_{S}, \omega\right)$, with $R\left(\mathbf{x}, \mathbf{x}_{S}, \omega\right)$ denoting the reflection response at $\partial \mathbb{D}_{0}$ of the medium below $\partial \mathbb{D}_{0}$. At $\partial \mathbb{D}_{A}$, we have $U_{B}^{ \pm}(\mathbf{x}, \omega)=G^{ \pm}\left(\mathbf{x}, \mathbf{x}_{S}, \omega\right)$. For state $A$ we consider a focal point at $\mathbf{x}_{A}=\left(x_{1, A}, x_{3, A}\right)$ at $\partial \mathbb{D}_{A}$. The medium in state $A$ is a truncated medium, which is identical to the actual medium between $\partial \mathbb{D}_{0}$ and $\partial \mathbb{D}_{A}$, and homogeneous below $\partial \mathbb{D}_{A}$. At $\partial \mathbb{D}_{0}$ a downgoing focusing function $U_{A}^{+}(\mathbf{x}, \omega)=f_{1}^{+}\left(\mathbf{x}, \mathbf{x}_{A}, \omega\right)$, with $\mathbf{x}=\left(x_{1}, x_{3,0}\right)$, is incident to the truncated medium. This function focuses at $\mathbf{x}_{A}$, hence, at $\partial \mathbb{D}_{A}$ we have $U_{A}^{+}(\mathbf{x}, \omega)=f_{1}^{+}\left(\mathbf{x}, \mathbf{x}_{A}, \omega\right)=\delta\left(x_{1}-x_{1, A}\right)$. The response to this focusing function at $\partial \mathbb{D}_{0}$ is $U_{A}^{-}(\mathbf{x}, \omega)=f_{1}^{-}\left(\mathbf{x}, \mathbf{x}_{A}, \omega\right)$. Because the truncated medium is homogeneous below $\partial \mathbb{D}_{A}$, we have $U_{A}^{-}(\mathbf{x}, \omega)=0$ at $\partial \mathbb{D}_{A}$. The quantities for both states are summarised in Table 2 .

Table 2: Quantities to derive equations (24) and (25).

\begin{tabular}{||l|c|c|c|c||}
\hline \hline & $U_{A}^{+}(\mathbf{x}, \omega)$ & $U_{A}^{-}(\mathbf{x}, \omega)$ & $U_{B}^{+}(\mathbf{x}, \omega)$ & $U_{B}^{-}(\mathbf{x}, \omega)$ \\
\hline $\mathbf{x}=\left(x_{1}, x_{3,0}\right)$ at $\partial \mathbb{D}_{0}$ & $f_{1}^{+}\left(\mathbf{x}, \mathbf{x}_{A}, \omega\right)$ & $f_{1}^{-}\left(\mathbf{x}, \mathbf{x}_{A}, \omega\right)$ & $\delta\left(x_{1}-x_{1, S}\right)$ & $R\left(\mathbf{x}, \mathbf{x}_{S}, \omega\right)$ \\
\hline $\mathbf{x}=\left(x_{1}, x_{3, A}\right)$ at $\partial \mathbb{D}_{A}$ & $\delta\left(x_{1}-x_{1, A}\right)$ & 0 & $G^{+}\left(\mathbf{x}, \mathbf{x}_{S}, \omega\right)$ & $G^{-}\left(\mathbf{x}, \mathbf{x}_{S}, \omega\right)$ \\
\hline \hline
\end{tabular}

Note that the downgoing focusing function $f_{1}^{+}\left(\mathbf{x}, \mathbf{x}_{A}, \omega\right)$, for $\mathbf{x}$ at $\partial \mathbb{D}_{0}$, is the inverse of the transmission response $T\left(\mathbf{x}_{A}, \mathbf{x}, \omega\right)$ of the truncated medium [31], hence

$$
f_{1}^{+}\left(\mathbf{x}, \mathbf{x}_{A}, \omega\right)=T^{\text {inv }}\left(\mathbf{x}_{A}, \mathbf{x}, \omega\right)
$$


for $\mathbf{x}$ at $\partial \mathbb{D}_{0}$. To avoid instabilities in the evanescent field, the focusing function is in practice spatially band-limited.

Substituting the quantities of Table 2 into equations (21) and $(22)$ gives

$$
G^{-}\left(\mathbf{x}_{A}, \mathbf{x}_{S}, \omega\right)+f_{1}^{-(\mathrm{c})}\left(\mathbf{x}_{S}, \mathbf{x}_{A}, \omega\right)=\int_{\partial \mathbb{D}_{0}} R\left(\mathbf{x}, \mathbf{x}_{S}, \omega\right) f_{1}^{+(\mathrm{c})}\left(\mathbf{x}, \mathbf{x}_{A}, \omega\right) \mathrm{d} \mathbf{x}
$$

and

$$
G^{+}\left(\mathbf{x}_{A}, \mathbf{x}_{S}, \omega\right)-\left\{f_{1}^{+}\left(\mathbf{x}_{S}, \mathbf{x}_{A}, \omega\right)\right\}^{*}=-\int_{\partial \mathbb{D}_{0}} R\left(\mathbf{x}, \mathbf{x}_{S}, \omega\right)\left\{f_{1}^{-}\left(\mathbf{x}, \mathbf{x}_{A}, \omega\right)\right\}^{*} \mathrm{~d} \mathbf{x}
$$

respectively. These are two representations for the upgoing and downgoing parts of the Green's function between $\mathbf{x}_{S}$ at the acquisition surface and $\mathbf{x}_{A}$ inside the non-reciprocal medium. They are expressed in terms of the reflection response $R\left(\mathbf{x}, \mathbf{x}_{S}, \omega\right)$ and a number of focusing functions. Unlike similar representations for reciprocal media [31, 78], the focusing functions in equation (24) are defined in the complementary version of the truncated medium. Therefore we cannot use the standard approach to retrieve the focusing functions and Green's functions from the reflection response $R\left(\mathbf{x}, \mathbf{x}_{S}, \omega\right)$. We obtain a second set of representations by replacing all quantities in equations (24) and (25) by the corresponding quantities in the complementary medium. For the focusing functions in equation (24) this implies they are replaced by their counterparts in the truncated actual medium. We thus obtain

$$
G^{-(\mathrm{c})}\left(\mathbf{x}_{A}, \mathbf{x}_{S}, \omega\right)+f_{1}^{-}\left(\mathbf{x}_{S}, \mathbf{x}_{A}, \omega\right)=\int_{\partial \mathbb{D}_{0}} R^{(\mathrm{c})}\left(\mathbf{x}, \mathbf{x}_{S}, \omega\right) f_{1}^{+}\left(\mathbf{x}, \mathbf{x}_{A}, \omega\right) \mathrm{d} \mathbf{x}
$$

and

$$
G^{+(\mathrm{c})}\left(\mathbf{x}_{A}, \mathbf{x}_{S}, \omega\right)-\left\{f_{1}^{+(\mathrm{c})}\left(\mathbf{x}_{S}, \mathbf{x}_{A}, \omega\right)\right\}^{*}=-\int_{\partial \mathbb{D}_{0}} R^{(\mathrm{c})}\left(\mathbf{x}, \mathbf{x}_{S}, \omega\right)\left\{f_{1}^{-(\mathrm{c})}\left(\mathbf{x}, \mathbf{x}_{A}, \omega\right)\right\}^{*} \mathrm{~d} \mathbf{x},
$$

respectively. Because in practical situations we do not have access to the reflection response $R^{(\mathrm{c})}\left(\mathbf{x}, \mathbf{x}_{S}, \omega\right)$ in the complementary medium, we derive a relation analogous to equation (13) for this reflection response. To this end, consider the quantities in Table 3, with $\mathbf{x}_{S}$ and $\mathbf{x}_{R}$ just above $\partial \mathbb{D}_{0}$, and with $\partial \mathbb{D}_{M}$ denoting a boundary below all inhomogeneities, so that there are no upgoing waves at $\partial \mathbb{D}_{M}$. Substituting the quantities of Table 3 into equation (21) (with $\partial \mathbb{D}_{A}$ replaced by $\partial \mathbb{D}_{M}$ ) gives

$$
R^{(\mathrm{c})}\left(\mathbf{x}_{R}, \mathbf{x}_{S}, \omega\right)=R\left(\mathbf{x}_{S}, \mathbf{x}_{R}, \omega\right) .
$$

Equations 24 - 27), with $R^{(\mathrm{c})}\left(\mathbf{x}, \mathbf{x}_{S}, \omega\right)$ replaced by $R\left(\mathbf{x}_{S}, \mathbf{x}, \omega\right)$, form the basis for the 
Marchenko method, discussed in the next section.

Table 3: Quantities to derive equation (28).

\begin{tabular}{||l|c|c|c|c||}
\hline \hline & $U_{A}^{+(\mathrm{c})}(\mathbf{x}, \omega)$ & $U_{A}^{-(\mathrm{c})}(\mathbf{x}, \omega)$ & $U_{B}^{+}(\mathbf{x}, \omega)$ & $U_{B}^{-}(\mathbf{x}, \omega)$ \\
\hline $\mathbf{x}=\left(x_{1}, x_{3,0}\right)$ at $\partial \mathbb{D}_{0}$ & $\delta\left(x_{1}-x_{1, S}\right)$ & $R^{(\mathrm{c})}\left(\mathbf{x}, \mathbf{x}_{S}, \omega\right)$ & $\delta\left(x_{1}-x_{1, R}\right)$ & $R\left(\mathbf{x}, \mathbf{x}_{R}, \omega\right)$ \\
\hline $\mathbf{x}=\left(x_{1}, x_{3, M}\right)$ at $\partial \mathbb{D}_{M}$ & $G^{+(\mathrm{c})}\left(\mathbf{x}, \mathbf{x}_{S}, \omega\right)$ & 0 & $G^{+}\left(\mathbf{x}, \mathbf{x}_{R}, \omega\right)$ & 0 \\
\hline \hline
\end{tabular}

\section{THE MARCHENKO METHOD FOR NON-RECIPROCAL MEDIA}

The standard multidimensional Marchenko method for reciprocal media [31, 78] uses the representations of equations (24) and (25), but without the superscript (c), to retrieve the focusing functions from the reflection response. Here we discuss how to modify this method for non-reciprocal media. We separate the representations of equations (24) - (27) into two sets, each set containing focusing functions in one and the same truncated medium. These sets are equations (25) and (26), with the focusing functions in the truncated actual medium, and equations (24) and (27), with the focusing functions in the truncated complementary medium. We start with the set of equations (25) and (26), which read in the time domain (using equation (28))

$$
G^{+}\left(\mathbf{x}_{A}, \mathbf{x}_{S}, t\right)-f_{1}^{+}\left(\mathbf{x}_{S}, \mathbf{x}_{A},-t\right)=-\int_{\partial \mathbb{D}_{0}} \mathrm{~d} \mathbf{x} \int_{-\infty}^{t} R\left(\mathbf{x}, \mathbf{x}_{S}, t-t^{\prime}\right) f_{1}^{-}\left(\mathbf{x}, \mathbf{x}_{A},-t^{\prime}\right) \mathrm{d} t^{\prime}
$$

and

$$
G^{-(\mathrm{c})}\left(\mathbf{x}_{A}, \mathbf{x}_{S}, t\right)+f_{1}^{-}\left(\mathbf{x}_{S}, \mathbf{x}_{A}, t\right)=\int_{\partial \mathbb{D}_{0}} \mathrm{~d} \mathbf{x} \int_{-\infty}^{t} R\left(\mathbf{x}_{S}, \mathbf{x}, t-t^{\prime}\right) f_{1}^{+}\left(\mathbf{x}, \mathbf{x}_{A}, t^{\prime}\right) \mathrm{d} t^{\prime}
$$

respectively. We introduce time windows to remove the Green's functions from these representations. Similar as in the reciprocal situation, we assume that the Green's function and the time-reversed focusing function on the left-hand side of equation (29) are separated in time, except for the direct arrivals [31. This is a reasonable assumption for media with smooth lateral variations, and for limited horizontal source-receiver distances. Let $t_{\mathrm{d}}\left(\mathbf{x}_{A}, \mathbf{x}_{S}\right)$ denote the travel time of the direct arrival of $G^{+}\left(\mathbf{x}_{A}, \mathbf{x}_{S}, t\right)$. We define a time window $w\left(\mathbf{x}_{A}, \mathbf{x}_{S}, t\right)=u\left(t_{\mathrm{d}}\left(\mathbf{x}_{A}, \mathbf{x}_{S}\right)-t_{\epsilon}-t\right)$, where $u(t)$ is the Heaviside function and $t_{\epsilon}$ a small positive time constant. Under the above-mentioned assumption, we have $w\left(\mathbf{x}_{A}, \mathbf{x}_{S}, t\right) G^{+}\left(\mathbf{x}_{A}, \mathbf{x}_{S}, t\right)=0$. For the focusing function on the left-hand side of equation 
(29) we write [31]

$$
\begin{aligned}
f_{1}^{+}\left(\mathbf{x}_{S}, \mathbf{x}_{A}, t\right) & =T^{\text {inv }}\left(\mathbf{x}_{A}, \mathbf{x}_{S}, t\right) \\
& =T_{\mathrm{d}}^{\text {inv }}\left(\mathbf{x}_{A}, \mathbf{x}_{S}, t\right)+M^{+}\left(\mathbf{x}_{S}, \mathbf{x}_{A}, t\right)
\end{aligned}
$$

where $T_{\mathrm{d}}^{\text {inv }}\left(\mathbf{x}_{A}, \mathbf{x}_{S}, t\right)$ is the inverse of the direct arrival of the transmission response of the truncated medium and $M^{+}\left(\mathbf{x}_{S}, \mathbf{x}_{A}, t\right)$ the scattering coda. The travel time of $T_{\mathrm{d}}^{\text {inv }}\left(\mathbf{x}_{A}, \mathbf{x}_{S}, t\right)$ is $-t_{\mathrm{d}}\left(\mathbf{x}_{A}, \mathbf{x}_{S}\right)$ and the scattering coda obeys $M^{+}\left(\mathbf{x}_{S}, \mathbf{x}_{A}, t\right)=0$ for $t \leq-t_{\mathrm{d}}\left(\mathbf{x}_{A}, \mathbf{x}_{S}\right)$. Hence, $w\left(\mathbf{x}_{A}, \mathbf{x}_{S}, t\right) f_{1}^{+}\left(\mathbf{x}_{S}, \mathbf{x}_{A},-t\right)=M^{+}\left(\mathbf{x}_{S}, \mathbf{x}_{A},-t\right)$. Applying the time window $w\left(\mathbf{x}_{A}, \mathbf{x}_{S}, t\right)$ to both sides of equation (29) thus yields

$$
M^{+}\left(\mathbf{x}_{S}, \mathbf{x}_{A},-t\right)=w\left(\mathbf{x}_{A}, \mathbf{x}_{S}, t\right) \int_{\partial \mathbb{D}_{0}} \mathrm{~d} \mathbf{x} \int_{-\infty}^{t} R\left(\mathbf{x}, \mathbf{x}_{S}, t-t^{\prime}\right) f_{1}^{-}\left(\mathbf{x}, \mathbf{x}_{A},-t^{\prime}\right) \mathrm{d} t^{\prime}
$$

Under the same conditions as those mentioned for equation (29), we assume that the Green's function and the focusing function in the left-hand side of equation (30) are separated in time (without overlap). Unlike for reciprocal media, we need a different time window to suppress the Green's function, because the latter is defined in the complementary medium. To this end we define a time window $w^{(\mathrm{c})}\left(\mathbf{x}_{A}, \mathbf{x}_{S}, t\right)=u\left(t_{\mathrm{d}}^{(\mathrm{c})}\left(\mathbf{x}_{A}, \mathbf{x}_{S}\right)-t_{\epsilon}-t\right)$, where $t_{\mathrm{d}}^{(\mathrm{c})}\left(\mathbf{x}_{A}, \mathbf{x}_{S}\right)$ denotes the travel time of the direct arrival in the complementary medium. Applying this window to both sides of equation (30) yields

$$
f_{1}^{-}\left(\mathbf{x}_{S}, \mathbf{x}_{A}, t\right)=w^{(\mathrm{c})}\left(\mathbf{x}_{A}, \mathbf{x}_{S}, t\right) \int_{\partial \mathbb{D}_{0}} \mathrm{~d} \mathbf{x} \int_{-\infty}^{t} R\left(\mathbf{x}_{S}, \mathbf{x}, t-t^{\prime}\right) f_{1}^{+}\left(\mathbf{x}, \mathbf{x}_{A}, t^{\prime}\right) \mathrm{d} t^{\prime} .
$$

Equations (32) and (33), with $f_{1}^{+}$given by equation (31), form a set of two equations for the two unknown functions $M^{+}\left(\mathbf{x}, \mathbf{x}_{A}, t\right)$ and $f_{1}^{-}\left(\mathbf{x}, \mathbf{x}_{A}, t\right)$ (with $\mathbf{x}$ at $\left.\partial \mathbb{D}_{0}\right)$. These functions can be resolved from equations (32) and (33), assuming $R\left(\mathbf{x}, \mathbf{x}_{S}, t\right), R\left(\mathbf{x}_{S}, \mathbf{x}, t\right), t_{\mathrm{d}}\left(\mathbf{x}_{A}, \mathbf{x}_{S}\right)$, $t_{\mathrm{d}}^{(\mathrm{c})}\left(\mathbf{x}_{A}, \mathbf{x}_{S}\right)$ and $T_{\mathrm{d}}^{\text {inv }}\left(\mathbf{x}_{A}, \mathbf{x}_{S}, t\right)$ are known for all $\mathbf{x}$ and $\mathbf{x}_{S}$ at $\partial \mathbb{D}_{0}$. The reflection responses $R\left(\mathbf{x}, \mathbf{x}_{S}, t\right)$ and $R\left(\mathbf{x}_{S}, \mathbf{x}, t\right)$ are obtained from measurements at the upper boundary $\partial \mathbb{D}_{0}$ of the medium. This involves deconvolution for the source function, decomposition and, when the upper boundary is a reflecting boundary, elimination of the surface-related multiple reflections [16]. Because the deconvolution is limited by the bandwidth of the source function, the time constant $t_{\epsilon}$ in the window function is taken equal to half the duration of the source function. This implies that the method will not account for short period multiples in layers with a thickness smaller than the wavelength [78]. The travel times $t_{\mathrm{d}}\left(\mathbf{x}_{A}, \mathbf{x}_{S}\right)$ and $t_{\mathrm{d}}^{(\mathrm{c})}\left(\mathbf{x}_{A}, \mathbf{x}_{S}\right)$, and the inverse of the direct arrival of the transmission response, $T_{\mathrm{d}}^{\text {inv }}\left(\mathbf{x}_{A}, \mathbf{x}_{S}, t\right)$, can be derived from a background model of the medium and its complementary version (once 
the background model is known, its complementary version follows immediately). A smooth background model is sufficient to derive these quantities, hence, no information about the scattering interfaces inside the medium is required. The iterative Marchenko scheme to solve for $M^{+}\left(\mathbf{x}, \mathbf{x}_{A}, t\right)$ and $f_{1}^{-}\left(\mathbf{x}, \mathbf{x}_{A}, t\right)$ reads

$$
\begin{aligned}
f_{1, k}^{-}\left(\mathbf{x}_{S}, \mathbf{x}_{A}, t\right) & =w^{(\mathrm{c})}\left(\mathbf{x}_{A}, \mathbf{x}_{S}, t\right) \int_{\partial \mathbb{D}_{0}} \mathrm{~d} \mathbf{x} \int_{-\infty}^{t} R\left(\mathbf{x}_{S}, \mathbf{x}, t-t^{\prime}\right) f_{1, k}^{+}\left(\mathbf{x}, \mathbf{x}_{A}, t^{\prime}\right) \mathrm{d} t^{\prime}, \\
M_{k+1}^{+}\left(\mathbf{x}_{S}, \mathbf{x}_{A},-t\right) & =w\left(\mathbf{x}_{A}, \mathbf{x}_{S}, t\right) \int_{\partial \mathbb{D}_{0}} \mathrm{~d} \mathbf{x} \int_{-\infty}^{t} R\left(\mathbf{x}, \mathbf{x}_{S}, t-t^{\prime}\right) f_{1, k}^{-}\left(\mathbf{x}, \mathbf{x}_{A},-t^{\prime}\right) \mathrm{d} t^{\prime},
\end{aligned}
$$

with

$$
f_{1, k}^{+}\left(\mathbf{x}, \mathbf{x}_{A}, t\right)=T_{\mathrm{d}}^{\mathrm{inv}}\left(\mathbf{x}_{A}, \mathbf{x}, t\right)+M_{k}^{+}\left(\mathbf{x}, \mathbf{x}_{A}, t\right),
$$

starting with $M_{0}^{+}\left(\mathbf{x}, \mathbf{x}_{A}, t\right)=0$. Once $M^{+}\left(\mathbf{x}, \mathbf{x}_{A}, t\right)$ and $f_{1}^{-}\left(\mathbf{x}, \mathbf{x}_{A}, t\right)$ are found, $f_{1}^{+}\left(\mathbf{x}, \mathbf{x}_{A}, t\right)$ is obtained from equation (31) and, subsequently, the Green's functions $G^{+}\left(\mathbf{x}_{A}, \mathbf{x}_{S}, t\right)$ and $G^{-(\mathrm{c})}\left(\mathbf{x}_{A}, \mathbf{x}_{S}, t\right)$ are obtained from equations 29$)$ and 30 . Note that only $G^{+}\left(\mathbf{x}_{A}, \mathbf{x}_{S}, t\right)$ is defined in the actual medium. To obtain $G^{-}\left(\mathbf{x}_{A}, \mathbf{x}_{S}, t\right)$ in the actual medium we consider the set of equations (24) and (27), which read in the time domain (using equation (28))

$$
G^{-}\left(\mathbf{x}_{A}, \mathbf{x}_{S}, t\right)+f_{1}^{-(\mathrm{c})}\left(\mathbf{x}_{S}, \mathbf{x}_{A}, t\right)=\int_{\partial \mathbb{D}_{0}} \mathrm{~d} \mathbf{x} \int_{-\infty}^{t} R\left(\mathbf{x}, \mathbf{x}_{S}, t-t^{\prime}\right) f_{1}^{+(\mathrm{c})}\left(\mathbf{x}, \mathbf{x}_{A}, t^{\prime}\right) \mathrm{d} t^{\prime}
$$

and

$$
G^{+(\mathrm{c})}\left(\mathbf{x}_{A}, \mathbf{x}_{S}, t\right)-f_{1}^{+(\mathrm{c})}\left(\mathbf{x}_{S}, \mathbf{x}_{A},-t\right)=-\int_{\partial \mathbb{D}_{0}} \mathrm{~d} \mathbf{x} \int_{-\infty}^{t} R\left(\mathbf{x}_{S}, \mathbf{x}, t-t^{\prime}\right) f_{1}^{-(\mathrm{c})}\left(\mathbf{x}, \mathbf{x}_{A},-t^{\prime}\right) \mathrm{d} t^{\prime}
$$

respectively. The same reasoning as above leads to the following iterative Marchenko scheme for the focusing functions in the truncated complementary medium

$$
\begin{aligned}
f_{1, k}^{-(\mathrm{c})}\left(\mathbf{x}_{S}, \mathbf{x}_{A}, t\right) & =w\left(\mathbf{x}_{A}, \mathbf{x}_{S}, t\right) \times \int_{\partial \mathbb{D}_{0}} \mathrm{~d} \mathbf{x} \int_{-\infty}^{t} R\left(\mathbf{x}, \mathbf{x}_{S}, t-t^{\prime}\right) f_{1, k}^{+(\mathrm{c})}\left(\mathbf{x}, \mathbf{x}_{A}, t^{\prime}\right) \mathrm{d} t^{\prime} \\
M_{k+1}^{+(\mathrm{c})}\left(\mathbf{x}_{S}, \mathbf{x}_{A},-t\right) & =w^{(\mathrm{c})}\left(\mathbf{x}_{A}, \mathbf{x}_{S}, t\right) \int_{\partial \mathbb{D}_{0}} \mathrm{~d} \mathbf{x} \int_{-\infty}^{t} R\left(\mathbf{x}_{S}, \mathbf{x}, t-t^{\prime}\right) f_{1, k}^{-(\mathrm{c})}\left(\mathbf{x}, \mathbf{x}_{A},-t^{\prime}\right) \mathrm{d} t^{\prime},
\end{aligned}
$$

with

$$
f_{1, k}^{+(\mathrm{c})}\left(\mathbf{x}, \mathbf{x}_{A}, t\right)=T_{\mathrm{d}}^{\mathrm{inv}(\mathrm{c})}\left(\mathbf{x}_{A}, \mathbf{x}, t\right)+M_{k}^{+(\mathrm{c})}\left(\mathbf{x}, \mathbf{x}_{A}, t\right)
$$

starting with $M_{0}^{+(\mathrm{c})}\left(\mathbf{x}, \mathbf{x}_{A}, t\right)=0$. Here $T_{\mathrm{d}}^{\mathrm{inv}(\mathrm{c})}\left(\mathbf{x}_{A}, \mathbf{x}, t\right)$ can be derived from the complemen- 
tary background model. Once the focusing functions $f_{1}^{+(\mathrm{c})}\left(\mathbf{x}, \mathbf{x}_{A}, t\right)$ and $f_{1}^{-(\mathrm{c})}\left(\mathbf{x}, \mathbf{x}_{A}, t\right)$ are found, the Green's functions $G^{-}\left(\mathbf{x}_{A}, \mathbf{x}_{S}, t\right)$ and $G^{+(\mathrm{c})}\left(\mathbf{x}_{A}, \mathbf{x}_{S}, t\right)$ are obtained from equations (37) and (38).

Table 4: Quantities to derive equation (42).

\begin{tabular}{||l|c|c|c|c||}
\hline \hline & $U_{A}^{+(\mathrm{c})}(\mathbf{x}, \omega)$ & $U_{A}^{-(\mathrm{c})}(\mathbf{x}, \omega)$ & $U_{B}^{+}(\mathbf{x}, \omega)$ & $U_{B}^{-}(\mathbf{x}, \omega)$ \\
\hline $\mathbf{x}=\left(x_{1}, x_{3, A}\right)$ at $\partial \mathbb{D}_{A}$ & $\delta\left(x_{1}-x_{1, A}\right)$ & $R^{(\mathrm{c})}\left(\mathbf{x}, \mathbf{x}_{A}, \omega\right)$ & $G^{+}\left(\mathbf{x}, \mathbf{x}_{S}, \omega\right)$ & $G^{-}\left(\mathbf{x}, \mathbf{x}_{S}, \omega\right)$ \\
\hline $\mathbf{x}=\left(x_{1}, x_{3, M}\right)$ at $\partial \mathbb{D}_{M}$ & $G^{+(\mathrm{c})}\left(\mathbf{x}, \mathbf{x}_{A}, \omega\right)$ & 0 & $G^{+}\left(\mathbf{x}, \mathbf{x}_{S}, \omega\right)$ & 0 \\
\hline \hline
\end{tabular}

We conclude this section by showing how $G^{+}\left(\mathbf{x}_{A}, \mathbf{x}_{S}, t\right)$ and $G^{-}\left(\mathbf{x}_{A}, \mathbf{x}_{S}, t\right)$ can be used to image the interior of the non-reciprocal medium. First we derive a mutual relation between these Green's functions. To this end, consider the quantities in Table 4 . Here $R^{(\mathrm{c})}\left(\mathbf{x}, \mathbf{x}_{A}, \omega\right)$ in state $A$ is the reflection response at $\partial \mathbb{D}_{A}$ of the complementary medium below $\partial \mathbb{D}_{A}$, with $\mathbf{x}_{A}$ defined just above $\partial \mathbb{D}_{A}$ and the medium in state $A$ being homogeneous above $\partial \mathbb{D}_{A}$. Substituting the quantities of Table 4 into equation $(21)$ (with $\partial \mathbb{D}_{0}$ and $\partial \mathbb{D}_{A}$ replaced by $\partial \mathbb{D}_{A}$ and $\partial \mathbb{D}_{M}$, respectively) and using equation $(28)$, gives

$$
G^{-}\left(\mathbf{x}_{A}, \mathbf{x}_{S}, \omega\right)=\int_{\partial \mathbb{D}_{A}} R\left(\mathbf{x}_{A}, \mathbf{x}, \omega\right) G^{+}\left(\mathbf{x}, \mathbf{x}_{S}, \omega\right) \mathrm{d} \mathbf{x}
$$

or, applying an inverse Fourier transformation to the time domain,

$$
G^{-}\left(\mathbf{x}_{A}, \mathbf{x}_{S}, t\right)=\int_{\partial \mathbb{D}_{A}} \mathrm{~d} \mathbf{x} \int_{-\infty}^{t} R\left(\mathbf{x}_{A}, \mathbf{x}, t-t^{\prime}\right) G^{+}\left(\mathbf{x}, \mathbf{x}_{S}, t^{\prime}\right) \mathrm{d} t^{\prime}
$$

Given the Green's functions $G^{+}\left(\mathbf{x}, \mathbf{x}_{S}, t\right)$ and $G^{-}\left(\mathbf{x}_{A}, \mathbf{x}_{S}, t\right)$ for all $\mathbf{x}_{A}$ and $\mathbf{x}$ at $\partial \mathbb{D}_{A}$ for a range of source positions $\mathbf{x}_{S}$ at $\partial \mathbb{D}_{0}$, the reflection response $R\left(\mathbf{x}_{A}, \mathbf{x}, t\right)$ for all $\mathbf{x}_{A}$ and $\mathbf{x}$ at $\partial \mathbb{D}_{A}$ can be resolved by multidimensional deconvolution [79 84]. An image can be obtained by selecting $R\left(\mathbf{x}_{A}, \mathbf{x}_{A}, t=0\right)$ and repeating the process for any $\mathbf{x}_{A}$ in the region of interest.

We discuss an alternative imaging approach for the special case of a laterally invariant medium. To this end we first rewrite equation 42 as a spatial convolution, taking $x_{1, S}=0$, hence

$$
G^{-}\left(x_{1, A}, x_{3, A}, x_{3, S}, \omega\right)=\int_{-\infty}^{\infty} R\left(x_{1, A}-x_{1}, x_{3, A}, \omega\right) G^{+}\left(x_{1}, x_{3, A}, x_{3, S}, \omega\right) \mathrm{d} x_{1} .
$$

We define the spatial Fourier transform of a function $P\left(x_{1}, x_{3}, \omega\right)$ as

$$
\tilde{P}\left(s_{1}, x_{3}, \omega\right)=\int_{-\infty}^{\infty} P\left(x_{1}, x_{3}, \omega\right) \exp \left(-i \omega s_{1} x_{1}\right) \mathrm{d} x_{1},
$$


(a)

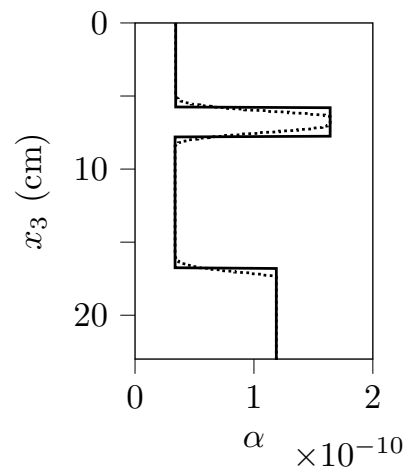

(d)

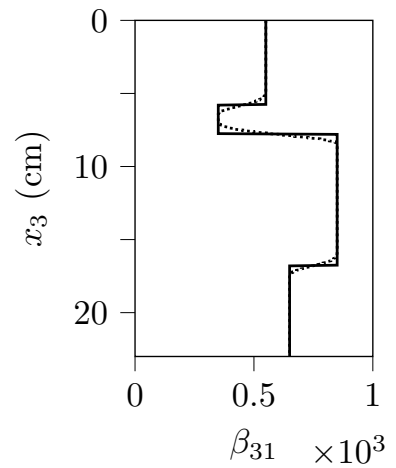

(b)

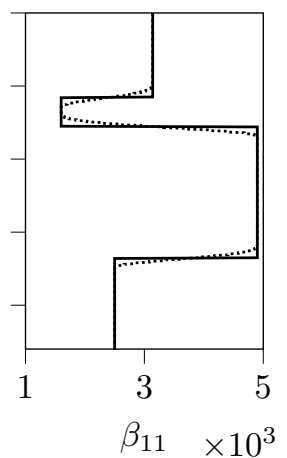

(e)

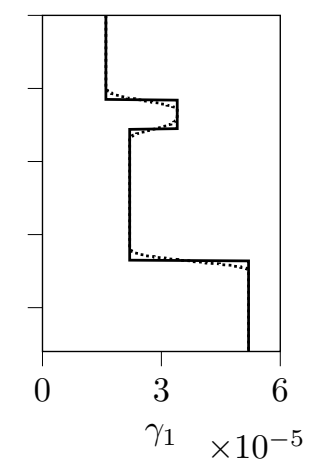

(c)

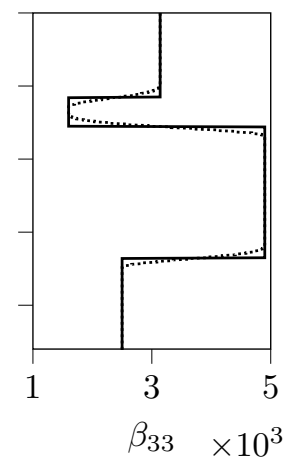

(f)

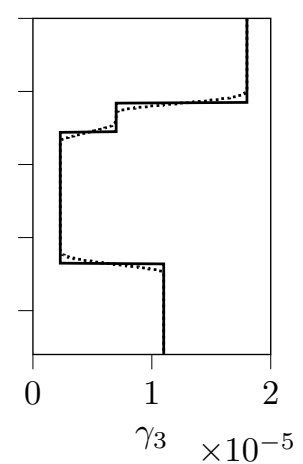

FIG. 2: Solid lines: parameters $\alpha\left(x_{3}\right), \beta_{11}\left(x_{3}\right), \beta_{33}\left(x_{3}\right), \beta_{31}\left(x_{3}\right), \gamma_{1}\left(x_{3}\right)$ and $\gamma_{3}\left(x_{3}\right)$ of the layered medium. Dotted lines: smoothed medium parameters, used to model the initial estimate of the focusing functions.

with $s_{1}$ being the horizontal slowness. In the $\left(s_{1}, x_{3}, \omega\right)$-domain, equation 44 becomes

$$
\tilde{G}^{-}\left(s_{1}, x_{3, A}, x_{3, S}, \omega\right)=\tilde{R}\left(s_{1}, x_{3, A}, \omega\right) \tilde{G}^{+}\left(s_{1}, x_{3, A}, x_{3, S}, \omega\right)
$$

or, applying an inverse Fourier transformation to the time domain,

$$
G^{-}\left(s_{1}, x_{3, A}, x_{3, S}, \tau\right)=\int_{-\infty}^{\tau} R\left(s_{1}, x_{3, A}, \tau-\tau^{\prime}\right) G^{+}\left(s_{1}, x_{3, A}, x_{3, S}, \tau^{\prime}\right) \mathrm{d} \tau^{\prime}
$$

Given the Green's functions $G^{+}\left(s_{1}, x_{3, A}, x_{3, S}, \tau\right)$ and $G^{-}\left(s_{1}, x_{3, A}, x_{3, S}, \tau\right)$, the reflection response $R\left(s_{1}, x_{3, A}, \tau\right)$ for each horizontal slowness $s_{1}$ can be resolved by $1 \mathrm{D}$ deconvolution. An image can be obtained by selecting $R\left(s_{1}, x_{3, A}, \tau=0\right)$ and repeating the process for all $s_{1}$ and for any $x_{3, A}$ in the region of interest. 


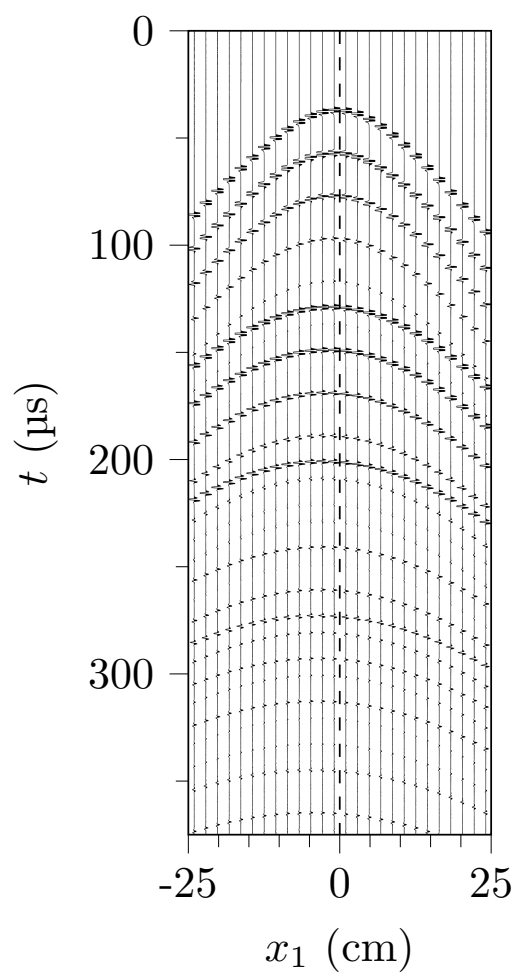

FIG. 3: The modeled reflection response $R\left(\mathbf{x}, \mathbf{x}_{S}, t\right) * S(t)$ at $\partial \mathbb{D}_{0}$. Note the asymmetry with respect to the dashed line due to the non-reciprocal medium parameters.

\section{NUMERICAL EXAMPLE}

We illustrate the proposed methodology with a numerical example, mimicking an ultrasound experiment. For simplicity we consider a horizontally layered medium, consisting of three homogeneous layers and a homogeneous half-space below the deepest layer. The medium parameters of the layered medium, $\alpha\left(x_{3}\right), \beta_{r s}\left(x_{3}\right)$ and $\gamma_{r}\left(x_{3}\right)$ are shown in Figure 2. In many practical situations the parameters $\beta_{31}\left(x_{3}\right)$ and $\gamma_{3}\left(x_{3}\right)$ will be zero, but we choose them to be non-zero to demonstrate the generality of the method. We define a source at $\mathbf{x}_{S}=(0,0)$ at the top of the first layer, which emits a time-symmetric wavelet $S(t)$ with a central frequency of $600 \mathrm{kHz}$ into the layered medium. We use a wavenumberfrequency domain modelling method [85], adjusted for non-reciprocal media, to model the response to this source. The modelled reflection response, $R\left(\mathbf{x}, \mathbf{x}_{S}, t\right) * S(t)$ at $\partial \mathbb{D}_{0}$ (the asterisk denoting convolution), is shown in Figure 3. To emphasise the multiple scattering, a time-dependent amplitude gain has been applied, using the function $\exp \{3 t / 375 \mu s\}$. Note that the apices of the reflection hyperbolae drift to the left with increasing time, which is a manifestation of the non-reciprocal medium parameters. Because the medium is laterally invariant, the response to any other source at the surface is just a laterally shifted version of the response in Figure 3. We apply the Marchenko method, discussed in detail in the 

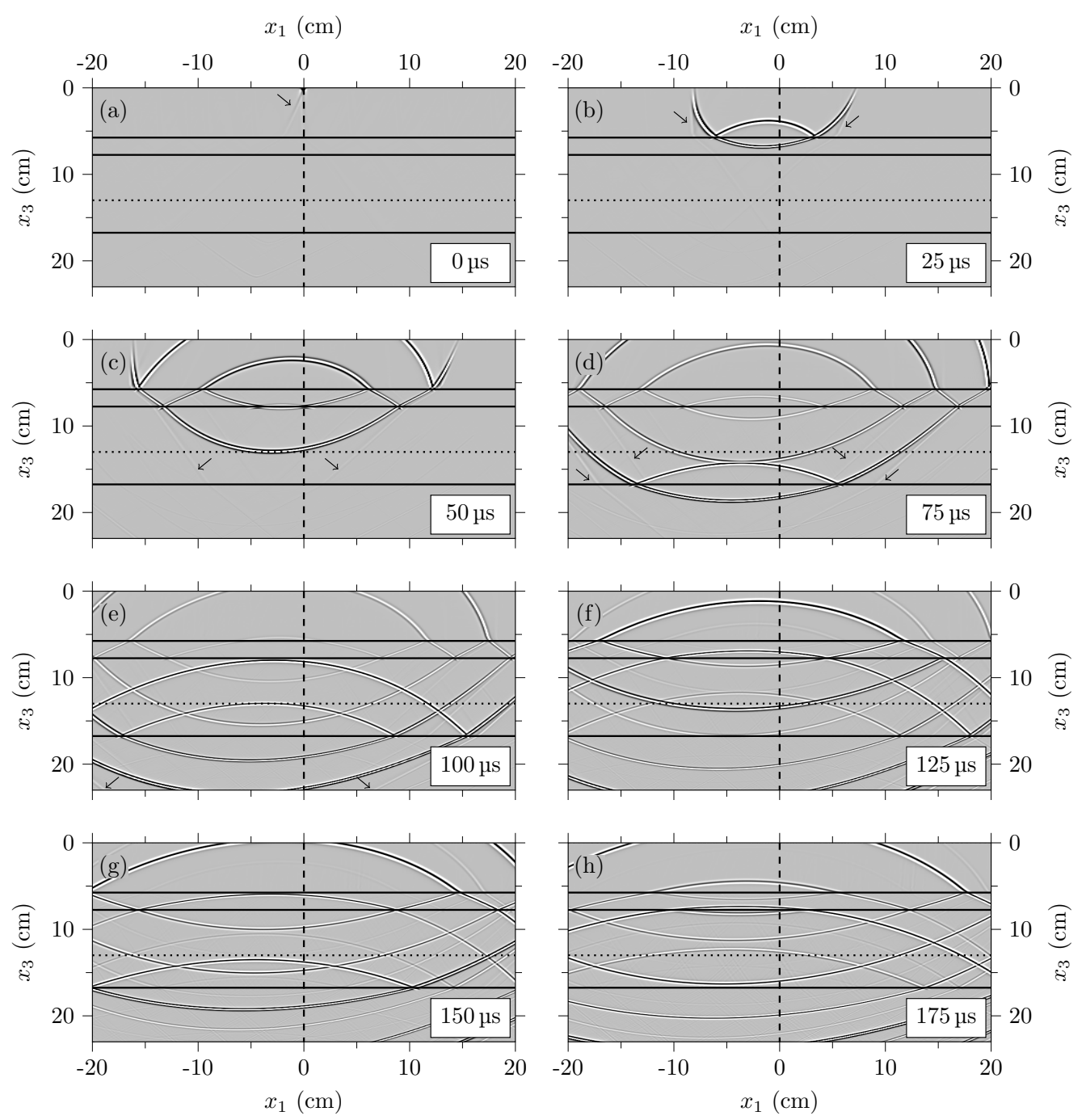

FIG. 4: Snapshots of $\left\{G^{+}\left(\mathbf{x}_{A}, \mathbf{x}_{S}, t\right)+G^{-}\left(\mathbf{x}_{A}, \mathbf{x}_{S}, t\right)\right\} * S(t)$, retrieved via equations 29] and (37), for $\mathbf{x}_{S}=(0,0)$ and variable $\mathbf{x}_{A}$.

previous section, to derive the focusing functions $f_{1}^{ \pm}\left(\mathbf{x}_{S}, \mathbf{x}_{A}, t\right)$ and $f_{1}^{ \pm(c)}\left(\mathbf{x}_{S}, \mathbf{x}_{A}, t\right)$ for fixed $\mathbf{x}_{S}=(0,0)$ and variable $\mathbf{x}_{A}$. As input we use the reflection response $R\left(\mathbf{x}, \mathbf{x}_{S}, t\right) * S(t)$ of the actual medium and the direct arrivals $T_{\mathrm{d}}\left(\mathbf{x}_{A}, \mathbf{x}, t\right)$ and $T_{\mathrm{d}}^{(\mathrm{c})}\left(\mathbf{x}_{A}, \mathbf{x}, t\right)$, modelled in a smoothed version of the truncated medium and its complementary version (the smoothed medium is indicated by the dotted lines in Figure 21). For simplicity we approximate the inverse direct arrivals $T_{\mathrm{d}}^{\text {inv }}\left(\mathbf{x}_{A}, \mathbf{x}, t\right)$ and $T_{\mathrm{d}}^{\text {inv(c) }}\left(\mathbf{x}_{A}, \mathbf{x}, t\right)$ in equations 36 and 41 by the time-reversals $T_{\mathrm{d}}\left(\mathbf{x}_{A}, \mathbf{x},-t\right)$ and $T_{\mathrm{d}}^{(\mathrm{c})}\left(\mathbf{x}_{A}, \mathbf{x},-t\right)$. For $t_{\epsilon}$ in the time windows $w\left(\mathbf{x}_{A}, \mathbf{x}_{S}, t\right)$ and $w^{(\mathrm{c})}\left(\mathbf{x}_{A}, \mathbf{x}_{S}, t\right)$ we choose half the duration of the symmetric wavelet $S(t)$, i.e., $t_{\epsilon}=0.65 \mu \mathrm{s}$, and the Heaviside functions are tapered. Because we consider a laterally invariant medium, the integrals in the right-hand sides of equations (34), (35), (39) and (40) are efficiently replaced by multiplications in the wavenumber-frequency domain. In total we apply 20 iterations of the 
(a)

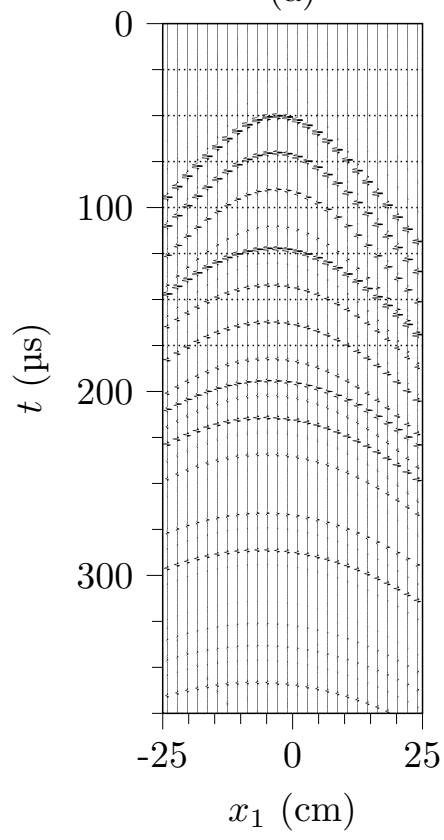

(b)

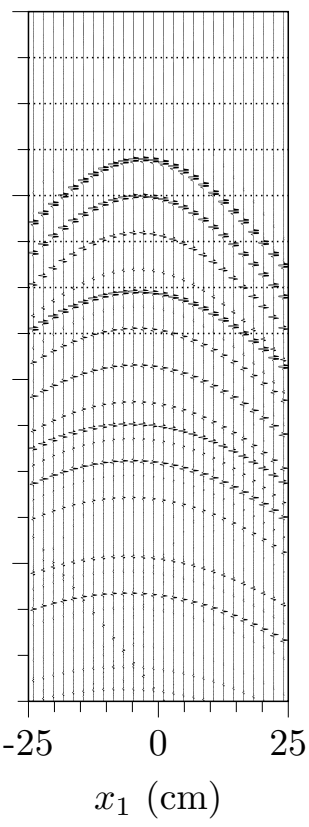

(c)

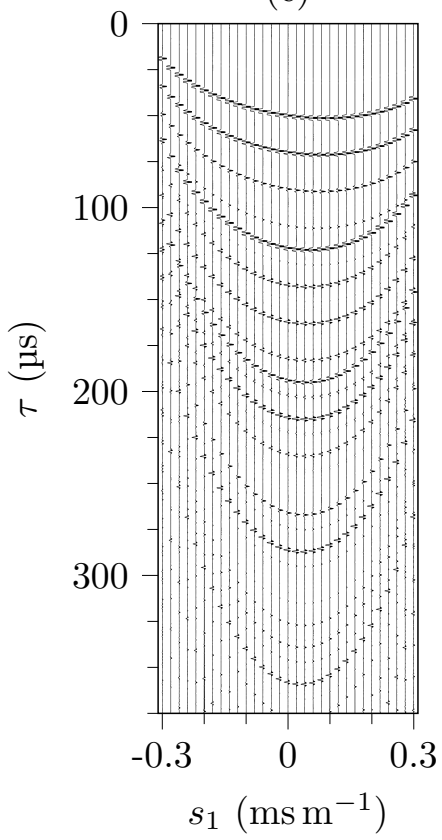

(d)

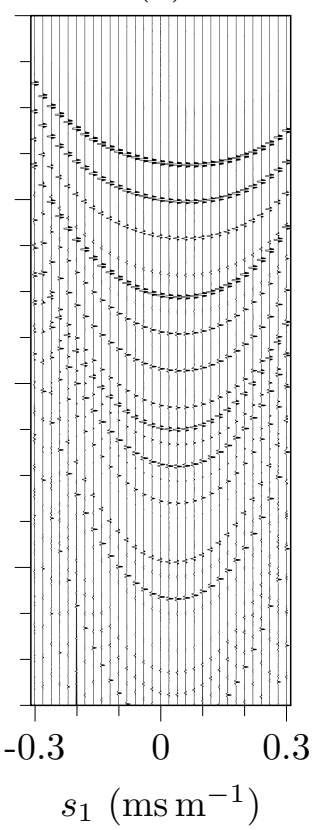

FIG. 5: Downgoing and upgoing wave fields at $x_{3, A}=13 \mathrm{~cm}$. (a) $G^{+}\left(x_{1}, x_{3, A}, x_{3, S}, t\right) * S(t)$, (b) $G^{-}\left(x_{1}, x_{3, A}, x_{3, S}, t\right) * S(t),(\mathrm{c}) G^{+}\left(s_{1}, x_{3, A}, x_{3, S}, \tau\right) * S(\tau),(\mathrm{d}) G^{-}\left(s_{1}, x_{3, A}, x_{3, S}, \tau\right) * S(\tau)$.

Marchenko scheme to derive the focusing functions $f_{1}^{ \pm}\left(\mathbf{x}_{S}, \mathbf{x}_{A}, t\right) * S(t)$ and the same number of iterations to derive $f_{1}^{ \pm(c)}\left(\mathbf{x}_{S}, \mathbf{x}_{A}, t\right) * S(t)$. These focusing functions are substituted into equations (29) and (37) (of which the integrals are also evaluated via the wavenumberfrequency domain) to obtain the wave fields $G^{+}\left(\mathbf{x}_{A}, \mathbf{x}_{S}, t\right) * S(t)$ and $G^{-}\left(\mathbf{x}_{A}, \mathbf{x}_{S}, t\right) * S(t)$. The superposition of these wave fields is shown in grey-level display in Figure 4 in the form of snapshots (i.e., wave fields at frozen time), for fixed $\mathbf{x}_{S}=(0,0)$ and variable $\mathbf{x}_{A}$. The amplitudes are clipped at $8 \%$ of the maximum amplitude. This figure clearly shows the propagation of the wave field from the source through the layered non-reciprocal medium. The wavefronts are asymmetric as a result of the non-reciprocal medium parameters (for a reciprocal medium these snapshots would be symmetric with respect to the vertical dashed lines). Multiple scattering between the layer interfaces is also clearly visible. The interfaces, indicated by the solid horizontal lines in each of the panels in Figure 4, are only shown here to aid the interpretation of the retrieved Green's functions. However, no explicit information of these interfaces has been used to retrieve these Green's functions; all information about the scattering at the layer interfaces comes directly from the reflection response $R\left(\mathbf{x}, \mathbf{x}_{S}, t\right) * S(t)$. The snapshots also exhibit some weak spurious linear events (indicated by the arrows in Figure 4), which are mainly caused by the negligence of evanescent waves and the absence of very large propagation angles in the reflection response.

Next, we image the interfaces of the layered medium, following the approach for a laterally 
(a)

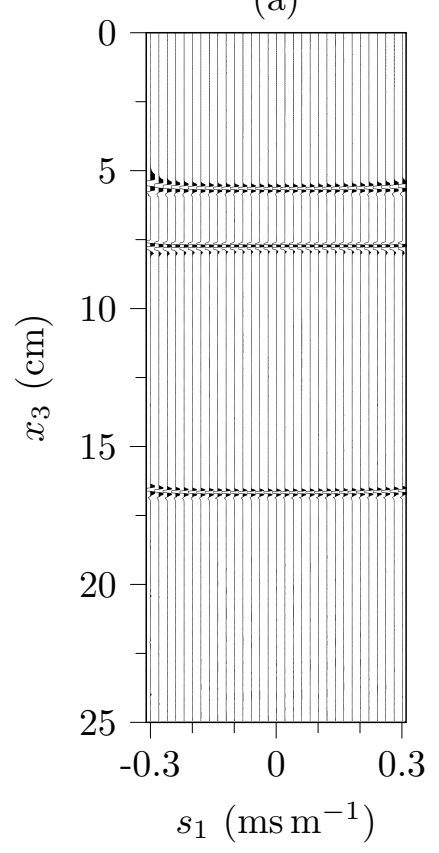

(b)

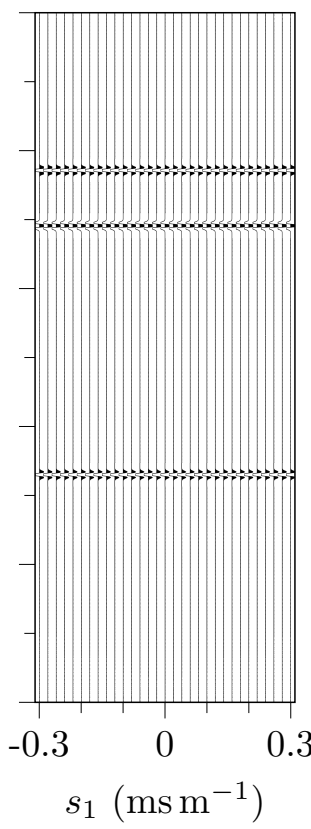

(c)

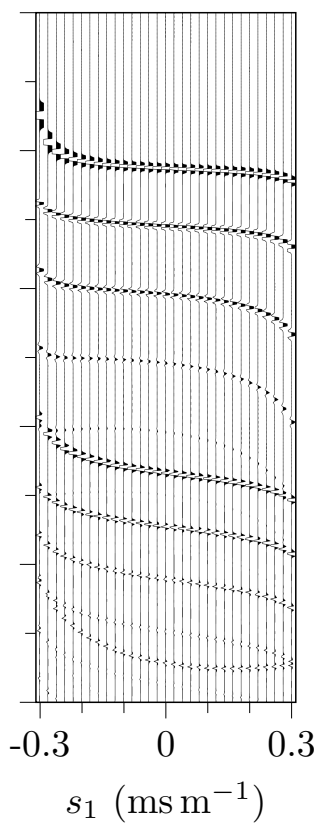

(d)

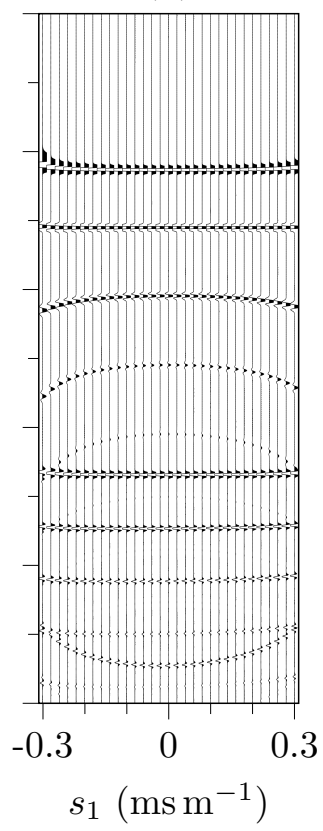

FIG. 6: Images in the $\left(s_{1}, x_{3}\right)$-domain of the layered medium of Figure2, (a) Marchenko imaging, accounting for non-reciprocity. (b) Reference reflectivity. (c) Primary imaging, ignoring non-reciprocity. (d) Primary imaging, accounting for non-reciprocity.

invariant medium described at the end of the previous section. Figures $5 \mathrm{a}, \mathrm{b}$ show the downgoing and upgoing wave fields $G^{+}\left(x_{1}, x_{3, A}, x_{3, S}, t\right) * S(t)$ and $G^{-}\left(x_{1}, x_{3, A}, x_{3, S}, t\right) * S(t)$, respectively, for $x_{3, A}=13 \mathrm{~cm}$ (the depth of the horizontal dotted lines in Figure 4). The horizontal dotted lines in Figures $5 \mathrm{a}, \mathrm{b}$ indicate the times of the snapshots in Figure 4. Figures 5r,d show the downgoing and upgoing wave fields $G^{+}\left(s_{1}, x_{3, A}, x_{3, S}, \tau\right) * S(\tau)$ and $G^{-}\left(s_{1}, x_{3, A}, x_{3, S}, \tau\right) * S(\tau)$, respectively, for a range of horizontal slownesses $s_{1}$. From these wave fields we derive the reflection response $R\left(s_{1}, x_{3, A}, \tau\right)$ by inverting equation 477 for each horizontal slowness $s_{1}$. The image at $x_{3, A}$ is obtained as $R\left(s_{1}, x_{3, A}, \tau=0\right)$. We repeat this for all $x_{3, A}$ between 0 and $25 \mathrm{~cm}$, in steps of $0.25 \mathrm{~mm}$. The result is shown in Figure 6a. This figure clearly shows images of the three interfaces in Figure 2. For comparison, Figure 6b shows, as a reference, the true reflectivity. The relative amplitude errors of the imaged interfaces are between $0.5 \%$ and $2 \%$, except for slownesses $\left|s_{1}\right|>0.2 \mathrm{~ms} / \mathrm{m}$, close to the evanescent field. Figure 6 c shows the result of standard primary imaging, ignoring non-reciprocity. The trace at $s_{1}=0$ contains images of the three interfaces at the correct depths, but it also contains false images caused by the internal multiples. Moreover, the traces for $s_{1} \neq 0$ contain images at wrong depths only. Finally, Figure 6 $\mathrm{d}$ is the result of primary imaging, taking non-reciprocity into account (by applying one iteration with our method). The three interfaces are imaged at the correct depths for all horizontal slownesses, 
but the false images are not suppressed.

\section{CONCLUSIONS}

Marchenko imaging has recently been introduced as a novel approach to account for multiple scattering in multidimensional acoustic and electromagnetic imaging. Given the recent interest in non-reciprocal materials, here we have extended the Marchenko approach for non-reciprocal media. We have derived two iterative Marchenko schemes, one to retrieve focusing functions in a truncated version of the actual medium and one to retrieve these functions in a truncated version of the complementary medium. Both schemes use the reflection response of the actual medium as input, plus estimates of the direct arrivals of the transmission response of the truncated actual medium (for the first scheme) and of the truncated complementary medium (for the second scheme). We have derived Green's function representations, which express the downgoing and upgoing part of the Green's function inside the non-reciprocal medium, in terms of the reflection response at the surface of the actual medium and the focusing functions in the truncated actual and complementary medium. From these downgoing and upgoing Green's functions, a reflectivity image of the medium can be obtained. We have illustrated the proposed approach at the hand of a numerical example for a horizontally layered non-reciprocal medium. This example shows an accurate wave field, propagating through the medium and scattering at its interfaces, retrieved from the reflection response at the surface. Moreover, it shows an accurately obtained artefact-free reflectivity image of the non-reciprocal medium, which confirms that the proposed method properly handles internal multiple scattering in a non-reciprocal medium.

\section{Acknowledgements}

We thank our colleague Evert Slob for his advise about electromagnetic waves in nonreciprocal media and reviewers Patrick Elison and Ivan Vasconcelos for their constructive comments, which helped to improve the paper. This work has received funding from the European Union's Horizon 2020 research and innovation programme: European Research Council (grant agreement 742703) and Marie Skłodowska-Curie (grant agreement 641943).

\section{Appendix A: Wave equations for non-reciprocal media}

We discuss wave equations for non-reciprocal media for (1) electromagnetic waves, (2) elastodynamic waves, and (3) acoustic waves. Next, (4) we derive a unified scalar wave 
equation for non-reciprocal media.

\section{Electromagnetic waves}

We start with the Maxwell equations for electromagnetic waves,

$$
\begin{aligned}
& \partial_{t} D_{i}-\epsilon_{i j k} \partial_{j} H_{k}=-J_{i}^{\mathrm{e}}, \\
& \partial_{t} B_{j}+\epsilon_{j k l} \partial_{k} E_{l}=-J_{j}^{\mathrm{m}} .
\end{aligned}
$$

Lower-case subscripts take the values 1, 2 and 3 and Einstein's summation convention applies to repeated subscripts. Exceptions are made for subscripts $r, s$ and $u$, which only take the values 1 and 3, and for subscript $t$, which denotes time. In equations (A1) and (A2), $E_{l}=$ $E_{l}(\mathbf{x}, t)$ is the electric field strength, $H_{k}=H_{k}(\mathbf{x}, t)$ the magnetic field strength, $D_{i}=D_{i}(\mathbf{x}, t)$ the electric flux density, $B_{j}=B_{j}(\mathbf{x}, t)$ the magnetic flux density, $J_{i}^{\mathrm{e}}=J_{i}^{\mathrm{e}}(\mathbf{x}, t)$ and $J_{j}^{\mathrm{m}}=$ $J_{j}^{\mathrm{m}}(\mathbf{x}, t)$ are source functions in terms of external electric and magnetic current densities and, finally, $\epsilon_{i j k}$ is the alternating tensor (or Levi-Civita tensor), with $\epsilon_{123}=\epsilon_{312}=\epsilon_{231}=1$, $\epsilon_{213}=\epsilon_{321}=\epsilon_{132}=-1$, and all other components being zero. For metamaterials, the field and source quantities in equations (A1) and (A2) are macroscopic quantities. These are sometimes denoted as $\left\langle H_{k}\right\rangle$ etc. [48], but for notational convenience we drop the brackets. In the low-frequency limit, the effective constitutive relations for lossless metamaterials read [48, 61, 86]

$$
\begin{aligned}
& D_{i}=\varepsilon_{i j} E_{j}+\eta_{i j} B_{j}, \\
& H_{k}=\theta_{k l} E_{l}+\nu_{k l} B_{l},
\end{aligned}
$$

where $\varepsilon_{i j}=\varepsilon_{i j}(\mathbf{x})$ is the permittivity, $\nu_{k l}=\nu_{k l}(\mathbf{x})$ the inverse permeability, and $\eta_{i j}=$ $\eta_{i j}(\mathbf{x})$ and $\theta_{k l}=\theta_{k l}(\mathbf{x})$ are coupling parameters. The inverse permeability is related to the permeability $\mu_{j k}=\mu_{j k}(\mathbf{x})$ via

$$
\mu_{j k} \nu_{k l}=\delta_{j l}
$$

with $\delta_{j l}$ the Kronecker delta function. The medium parameters in equations (A3) and (A4) are effective parameters. In general they are anisotropic, even when they are isotropic at micro scale. For a non-reciprocal lossless metamaterial they are real-valued and obey the following symmetry relations [61, 62, 65]

$$
\varepsilon_{i j}=\varepsilon_{j i}, \quad \nu_{k l}=\nu_{l k}, \quad \mu_{j k}=\mu_{k j}, \quad \eta_{i j}=-\theta_{j i}
$$


We reorganise the constitutive relations into a set of explicit expressions for $D_{i}$ and $B_{j}$. To this end we multiply both sides of equation (A4) by $\mu_{j k}$. Using equation (A5) this gives

$$
B_{j}=-\mu_{j k} \theta_{k l} E_{l}+\mu_{j k} H_{k}
$$

Substitution into equation A3 gives

$$
D_{i}=\left(\varepsilon_{i l}-\eta_{i j} \mu_{j k} \theta_{k l}\right) E_{l}+\eta_{i j} \mu_{j k} H_{k}
$$

Equations (A8) and (A7) form a new set of effective constitutive relations [63, 87,

$$
\begin{aligned}
& D_{i}=\varepsilon_{i l}^{o} E_{l}+\xi_{i k} H_{k}, \\
& B_{j}=\zeta_{j l} E_{l}+\mu_{j k} H_{k},
\end{aligned}
$$

with

$$
\begin{aligned}
\varepsilon_{i l}^{o} & =\varepsilon_{i l}-\eta_{i j} \mu_{j k} \theta_{k l} \\
\xi_{i k} & =\eta_{i j} \mu_{j k} \\
\zeta_{j l} & =-\mu_{j k} \theta_{k l} .
\end{aligned}
$$

On account of equation $(\mathrm{A} 6)$, these parameters obey the following symmetry relations [61, 88]

$$
\varepsilon_{i l}^{o}=\varepsilon_{l i}^{o}, \quad \xi_{l j}=\zeta_{j l} .
$$

Substitution of constitutive relations (A9) and (A10) into Maxwell equations (A1) and (A2), using $\xi_{l j}=\zeta_{j l}$, gives

$$
\begin{aligned}
& \varepsilon_{i l}^{o} \partial_{t} E_{l}+\xi_{i k} \partial_{t} H_{k}-\epsilon_{i j k} \partial_{j} H_{k}=-J_{i}^{\mathrm{e}} \\
& \xi_{l j} \partial_{t} E_{l}+\mu_{j k} \partial_{t} H_{k}+\epsilon_{j k l} \partial_{k} E_{l}=-J_{j}^{\mathrm{m}} .
\end{aligned}
$$

Next, we assume that the wave fields, sources and medium parameters are independent of the $x_{2}$-coordinate. Furthermore, we assume $\varepsilon_{21}^{o}=\varepsilon_{23}^{o}=0, \mu_{21}=\mu_{23}=0, \xi_{11}=\xi_{22}=$ $\xi_{33}=\xi_{13}=\xi_{31}=0$. Then equation A15 for $i=1,2,3$ (using $\varepsilon_{13}^{o}=\varepsilon_{31}^{o}$ ) and equation A16) for $j=1,2,3$ (using $\mu_{13}=\mu_{31}$ ) yield six equations, describing wave propagation in the $\left(x_{1}, x_{3}\right)$-plane. These can be separated into two independent sets of equations, for transverse-electric (TE) waves (with wave field quantities $E_{2}, H_{1}$ and $H_{3}$ ) and for transversemagnetic (TM) waves (with wave field quantities $H_{2}, E_{1}$ and $E_{3}$ ). For TE wave propagation 
in the $\left(x_{1}, x_{3}\right)$-plane we thus obtain

$$
\begin{aligned}
& \varepsilon_{22}^{o} \partial_{t} E_{2}+\xi_{21} \partial_{t} H_{1}+\xi_{23} \partial_{t} H_{3}+\partial_{1} H_{3}-\partial_{3} H_{1}=-J_{2}^{\mathrm{e}}, \\
& \mu_{11} \partial_{t} H_{1}+\mu_{31} \partial_{t} H_{3}+\xi_{21} \partial_{t} E_{2}-\partial_{3} E_{2}=-J_{1}^{\mathrm{m}}, \\
& \mu_{31} \partial_{t} H_{1}+\mu_{33} \partial_{t} H_{3}+\xi_{23} \partial_{t} E_{2}+\partial_{1} E_{2}=-J_{3}^{\mathrm{m}}
\end{aligned}
$$

and for TM wave propagation in the $\left(x_{1}, x_{3}\right)$-plane

$$
\begin{aligned}
& \mu_{22} \partial_{t} H_{2}+\xi_{12} \partial_{t} E_{1}+\xi_{32} \partial_{t} E_{3}-\partial_{1} E_{3}+\partial_{3} E_{1}=-J_{2}^{\mathrm{m}}, \\
& \varepsilon_{11}^{o} \partial_{t} E_{1}+\varepsilon_{31}^{o} \partial_{t} E_{3}+\xi_{12} \partial_{t} H_{2}+\partial_{3} H_{2}=-J_{1}^{\mathrm{e}}, \\
& \varepsilon_{31}^{o} \partial_{t} E_{1}+\varepsilon_{33}^{o} \partial_{t} E_{3}+\xi_{32} \partial_{t} H_{2}-\partial_{1} H_{2}=-J_{3}^{\mathrm{e}} .
\end{aligned}
$$

\section{Elastodynamic waves}

We start with the equilibrium of momentum [56] and the deformation equation [59]

$$
\begin{aligned}
& \partial_{t} m_{i}-\partial_{j} \tau_{i j}=F_{i}, \\
& \partial_{t} e_{k l}-\frac{1}{2}\left(\partial_{k} v_{l}+\partial_{l} v_{k}\right)=-h_{k l} .
\end{aligned}
$$

Here $m_{i}=m_{i}(\mathbf{x}, t)$ is the momentum density, $\tau_{i j}=\tau_{i j}(\mathbf{x}, t)$ the stress tensor, $e_{k l}=e_{k l}(\mathbf{x}, t)$ the strain tensor, $v_{k}=v_{k}(\mathbf{x}, t)$ the particle velocity and $F_{i}=F_{i}(\mathbf{x}, t)$ and $h_{k l}=h_{k l}(\mathbf{x}, t)$ are source functions in terms of external force and deformation-rate density. For metamaterials, the field and source quantities in equations (A23) and (A24) are macroscopic quantities. These are sometimes denoted as $\left\langle\tau_{i j}\right\rangle$ etc. [51], but for notational convenience we drop the brackets. They obey the following symmetry relations

$$
\tau_{i j}=\tau_{j i}, \quad e_{k l}=e_{l k}, \quad h_{k l}=h_{l k} .
$$

In the low-frequency limit, the effective constitutive relations for metamaterials read [51, 52, 56 ]

$$
\begin{aligned}
& m_{i}=\rho_{i k} v_{k}+S_{i k l}^{(2)} e_{k l}, \\
& \tau_{m n}=S_{m n p}^{(1)} v_{p}+c_{m n p q} e_{p q},
\end{aligned}
$$

where $\rho_{i k}=\rho_{i k}(\mathbf{x})$ is the mass density tensor, $c_{m n p q}=c_{m n p q}(\mathbf{x})$ the stiffness tensor and $S_{m n p}^{(1)}=S_{m n p}^{(1)}(\mathbf{x})$ and $S_{i k l}^{(2)}=S_{i k l}^{(2)}(\mathbf{x})$ are coupling parameters. The stiffness tensor is related 
to the compliance tensor $s_{k l m n}=s_{k l m n}(\mathbf{x})$ via

$$
s_{k l m n} c_{m n p q}=\frac{1}{2}\left(\delta_{k p} \delta_{l q}+\delta_{k q} \delta_{l p}\right)
$$

The medium parameters in equations A26 and A27) are effective parameters. In general they are anisotropic, even when they are isotropic at micro scale. An example of a non-reciprocal metamaterial is a phononic crystal of which the stiffness and mass density are modulated in a wave-like fashion [56. For this situation, equations A26) and (A27) are defined in a coordinate system that moves along with the modulating wave, so that the effective medium parameters in this coordinate system are time-independent. For a non-reciprocal lossless metamaterial the medium parameters are real-valued and obey the following symmetry relations [56]

$$
\begin{aligned}
& \rho_{i k}=\rho_{k i}, \\
& c_{m n p q}=c_{n m p q}=c_{m n q p}=c_{p q m n}, \\
& s_{k l m n}=s_{l k m n}=s_{k l n m}=s_{m n k l}, \\
& S_{m n p}^{(1)}=S_{n m p}^{(1)}, \\
& S_{i k l}^{(2)}=S_{i l k}^{(2)} \\
& S_{i k l}^{(2)}=-S_{k l i}^{(1)} .
\end{aligned}
$$

We reorganise the constitutive relations into a set of explicit expressions for $m_{i}$ and $e_{k l}$. To this end we multiply both sides of equation A27) by $s_{k l m n}$. Using equations (A28) and $e_{k l}=e_{l k}$ this gives

$$
e_{k l}=-s_{k l m n} S_{m n p}^{(1)} v_{p}+s_{k l m n} \tau_{m n}
$$

Substitution into equation $(\mathrm{A} 26)$ gives

$$
m_{i}=\left(\rho_{i p}-S_{i k l}^{(2)} s_{k l m n} S_{m n p}^{(1)}\right) v_{p}+S_{i k l}^{(2)} s_{k l m n} \tau_{m n}
$$

Equations A36 and A35 form a new set of effective constitutive relations,

$$
\begin{aligned}
& m_{i}=\rho_{i p}^{o} v_{p}-\xi_{i m n} \tau_{m n}, \\
& e_{k l}=-\zeta_{k l p} v_{p}+s_{k l m n} \tau_{m n},
\end{aligned}
$$


with

$$
\begin{aligned}
& \rho_{i p}^{o}=\rho_{i p}-S_{i k l}^{(2)} s_{k l m n} S_{m n p}^{(1)}, \\
& \xi_{i m n}=-S_{i k l}^{(2)} s_{k l m n}, \\
& \zeta_{k l p}=s_{k l m n} S_{m n p}^{(1)} .
\end{aligned}
$$

For convenience we use the same symbols $(\xi$ and $\zeta$ ) for the coupling parameters as in the electromagnetic constitutive relations, but of course these are different quantities with different physical dimensions. On account of equations A29, A31) and A34 these parameters obey the following symmetry relations

$$
\rho_{i p}^{o}=\rho_{p i}^{o}, \quad \zeta_{k l p}=\zeta_{l k p}, \quad \xi_{i m n}=\xi_{i n m}, \quad \xi_{p k l}=\zeta_{k l p} .
$$

Substitution of constitutive relations (A37) and (A38) into equations (A23) and (A24), using $\xi_{p k l}=\zeta_{k l p}$, gives

$$
\begin{aligned}
& \rho_{i p}^{o} \partial_{t} v_{p}-\xi_{i m n} \partial_{t} \tau_{m n}-\partial_{j} \tau_{i j}=F_{i} \\
& -\xi_{p k l} \partial_{t} v_{p}+s_{k l m n} \partial_{t} \tau_{m n}-\frac{1}{2}\left(\partial_{k} v_{l}+\partial_{l} v_{k}\right)=-h_{k l} .
\end{aligned}
$$

Next, we assume that the wave fields, sources and medium parameters are independent of the $x_{2}$-coordinate. Furthermore, we assume $\rho_{21}^{o}=\rho_{23}^{o}=0, s_{1211}=s_{1222}=s_{1233}=$ $s_{1213}=s_{3211}=s_{3222}=s_{3233}=s_{3213}=0$ and $\xi_{112}=\xi_{132}=\xi_{211}=\xi_{222}=\xi_{233}=\xi_{213}=$ $\xi_{312}=\xi_{332}=0$. Then equation $\mathrm{A} 43$ ) for $i=2$ (using $\xi_{2 m n}=\xi_{2 n m}$ and $\tau_{m n}=\tau_{n m}$ ) and equation (A44) for $k=1,3$ (setting $l=2$ in both cases and using equation (A31) yield three equations, describing the propagation of horizontally-polarised shear ( $\mathrm{SH}$ ) waves (with wave field quantities $v_{2}, \tau_{21}$ and $\left.\tau_{23}\right)$ in the $\left(x_{1}, x_{3}\right)$-plane:

$$
\begin{aligned}
& \rho_{22}^{o} \partial_{t} v_{2}-2 \xi_{221} \partial_{t} \tau_{21}-2 \xi_{223} \partial_{t} \tau_{23}-\partial_{1} \tau_{21}-\partial_{3} \tau_{23}=F_{2} \\
& -4 s_{1221} \partial_{t} \tau_{21}-4 s_{1223} \partial_{t} \tau_{23}+2 \xi_{221} \partial_{t} v_{2}+\partial_{1} v_{2}=2 h_{21} \\
& -4 s_{1223} \partial_{t} \tau_{21}-4 s_{3223} \partial_{t} \tau_{23}+2 \xi_{223} \partial_{t} v_{2}+\partial_{3} v_{2}=2 h_{23}
\end{aligned}
$$




\section{Acoustic waves}

We derive the equations for acoustic waves from those for elastodynamic waves. To this end we make the following substitutions

$$
\begin{aligned}
\tau_{i j} & =-\delta_{i j} p, \\
e_{k l} & =\frac{1}{3} \delta_{k l} \Theta, \\
h_{k l} & =\frac{1}{3} \delta_{k l} q, \\
c_{m n p q} & =\delta_{m n} \delta_{p q} K .
\end{aligned}
$$

Here $p=p(\mathbf{x}, t)$ is the acoustic pressure, $\Theta=\Theta(\mathbf{x}, t)$ the cubic dilatation, $q=q(\mathbf{x}, t)$ a source function in terms of volume injection-rate density and $K=K(\mathbf{x})$ the effective bulk modulus of the medium. With these substitutions, equations (A23) and A24 become

$$
\begin{aligned}
& \partial_{t} m_{i}+\partial_{i} p=F_{i} \\
& \frac{1}{3} \delta_{k l} \partial_{t} \Theta-\frac{1}{2}\left(\partial_{k} v_{l}+\partial_{l} v_{k}\right)=-\frac{1}{3} \delta_{k l} q
\end{aligned}
$$

Multiplying both sides of the latter equation by $\delta_{k l}$ we obtain

$$
\partial_{t} \Theta-\partial_{k} v_{k}=-q
$$

Similarly, the constitutive relations A26 and A27 become

$$
\begin{aligned}
& m_{i}=\rho_{i k} v_{k}+\frac{1}{3} S_{i l l}^{(2)} \Theta \\
& -\delta_{m n} p=S_{m n p}^{(1)} v_{p}+\frac{1}{3} \delta_{m n} \delta_{p q} K \delta_{p q} \Theta
\end{aligned}
$$

Multiplying both sides of the latter equation by $\frac{1}{3} \delta_{m n}$ we obtain

$$
-p=\frac{1}{3} S_{m m p}^{(1)} v_{p}+K \Theta
$$

On account of equations (A29) and A34, the effective medium parameters in constitutive relations A55 and A57) obey the following symmetry relations

$$
\rho_{i k}=\rho_{k i}, \quad S_{i l l}^{(2)}=-S_{m m i}^{(1)} .
$$


We reorganise the constitutive relations into a set of explicit expressions for $m_{i}$ and $\Theta$. To this end we divide both sides of equation A57) by $K$, which gives

$$
\Theta=-\zeta_{p} v_{p}-\kappa p
$$

with

$$
\begin{aligned}
& \zeta_{p}=\frac{1}{3} \kappa S_{m m p}^{(1)}, \\
& \kappa=1 / K .
\end{aligned}
$$

Substitution into equation $\mathrm{A} 55$ gives

$$
m_{i}=\rho_{i p}^{o} v_{p}+\xi_{i} p
$$

with

$$
\begin{aligned}
& \rho_{i p}^{o}=\rho_{i p}-\frac{1}{9} \kappa S_{i l l}^{(2)} S_{m m p}^{(1)}, \\
& \xi_{i}=-\frac{1}{3} \kappa S_{i l l}^{(2)}
\end{aligned}
$$

Equations A62 and A59 form a new set of constitutive relations. On account of equation (A58), the medium parameters in these relations obey the following symmetry relations

$$
\rho_{i p}^{o}=\rho_{p i}^{o}, \quad \xi_{p}=\zeta_{p}
$$

Substitution of constitutive relations (A62) and A59 into equations (A52) and (A54), using $\xi_{p}=\zeta_{p}$, gives

$$
\begin{aligned}
& \rho_{i p}^{o} \partial_{t} v_{p}+\xi_{i} \partial_{t} p+\partial_{i} p=F_{i}, \\
& \xi_{p} \partial_{t} v_{p}+\kappa \partial_{t} p+\partial_{k} v_{k}=q .
\end{aligned}
$$

Next, we assume that the wave fields, sources and medium parameters are independent of the $x_{2}$-coordinate. Furthermore, we assume $\rho_{12}^{o}=\rho_{32}^{o}=0$ and $\xi_{2}=0$. Then equation A66) for $i=1,3$ (using $\rho_{13}^{o}=\rho_{31}^{o}$ ) and equation (A67) yield three equations, describing the propagation of acoustic (AC) waves (with wave field quantities $p, v_{1}$ and $v_{3}$ ) in the 
$\left(x_{1}, x_{3}\right)$-plane:

$$
\begin{aligned}
& \kappa \partial_{t} p+\xi_{1} \partial_{t} v_{1}+\xi_{3} \partial_{t} v_{3}+\partial_{1} v_{1}+\partial_{3} v_{3}=q, \\
& \rho_{11}^{o} \partial_{t} v_{1}+\rho_{31}^{o} \partial_{t} v_{3}+\xi_{1} \partial_{t} p+\partial_{1} p=F_{1}, \\
& \rho_{31}^{o} \partial_{t} v_{1}+\rho_{33}^{o} \partial_{t} v_{3}+\xi_{3} \partial_{t} p+\partial_{3} p=F_{3} .
\end{aligned}
$$

\section{Unified scalar wave equation}

The systems of equations for transverse-electric waves (TE waves, equations A17 (A19), transverse-magnetic waves (TM waves, equations (A20 - A22), horizontally polarised shear waves (SH waves, equations A45 - A47) ) and acoustic waves (AC waves, equations $\mathrm{A} 68 \mathrm{~A}-\mathrm{A} 70)$, can all be cast in the following form

$$
\begin{aligned}
& \alpha \partial_{t} P+\left(\partial_{r}+\gamma_{r} \partial_{t}\right) Q_{r}=B, \\
& \left(\partial_{s}+\gamma_{s} \partial_{t}\right) P+\beta_{s u} \partial_{t} Q_{u}=C_{s},
\end{aligned}
$$

with $\beta_{s u}=\beta_{u s}$. Recall that subscripts $r, s$ and $u$ only take the values 1 and 3 . The field quantities, medium parameters and source functions in these equations are given in Table 1 for TE, TM, SH and AC waves. We derive a scalar wave equation for $P$ by eliminating $Q_{r}$ from equations $\mathrm{A} 71$ and $\mathrm{A} 72$. We define the inverse of $\beta_{s u}$ via

$$
\vartheta_{r s} \beta_{s u}=\delta_{r u}
$$

Because $\beta_{s u}$ is a symmetric $2 \times 2$ tensor, the following simple expressions hold for $\vartheta_{\text {rs }}$

$$
\begin{aligned}
\vartheta_{11} & =\beta_{33} / \Delta, \\
\vartheta_{13}=\vartheta_{31} & =-\beta_{31} / \Delta, \\
\vartheta_{33} & =\beta_{11} / \Delta,
\end{aligned}
$$

with

$$
\Delta=\beta_{11} \beta_{33}-\beta_{31}^{2}
$$

Apply $\partial_{t}$ to both sides of equation A71 and $\left(\partial_{r}+\gamma_{r} \partial_{t}\right) \vartheta_{r s}$ to both sides of equation A72 and subtract the results. Using the fact that the effective medium parameters are time- 
independent, this gives

$$
\left(\partial_{r}+\gamma_{r} \partial_{t}\right) \vartheta_{r s}\left(\partial_{s}+\gamma_{s} \partial_{t}\right) P-\alpha \partial_{t}^{2} P=\left(\partial_{r}+\gamma_{r} \partial_{t}\right) \vartheta_{r s} C_{s}-\partial_{t} B
$$

\section{Appendix B: Decomposition of the reciprocity theorems for non-reciprocal media}

We derive (1) a unified matrix-vector wave equation for non-reciprocal media, (2) apply decomposition to the operator matrix, and (3) use the symmetry properties of the decomposed operators to derive reciprocity theorems for decomposed wave fields.

\section{Unified matrix-vector wave equation}

Using the Fourier transform, defined in equation (7), we transform equations (A71) and A72 to the space-frequency domain, yielding

$$
\begin{aligned}
& -i \omega \alpha P+\left(\partial_{r}-i \omega \gamma_{r}\right) Q_{r}=B \\
& \left(\partial_{s}-i \omega \gamma_{s}\right) P-i \omega \beta_{s u} Q_{u}=C_{s}
\end{aligned}
$$

We derive a matrix-vector wave equation of the form

$$
\partial_{3} \mathbf{q}=\mathcal{A} \mathbf{q}+\mathbf{d}
$$

with wave vector $\mathbf{q}=\mathbf{q}(\mathbf{x}, \omega)$ and source vector $\mathbf{d}=\mathbf{d}(\mathbf{x}, \omega)$ defined as

$$
\mathbf{q}=\left(\begin{array}{c}
P \\
Q_{3}
\end{array}\right), \quad \mathbf{d}=\left(\begin{array}{c}
C^{o} \\
B^{o}
\end{array}\right)
$$

and operator matrix $\mathcal{A}=\mathcal{A}(\mathbf{x}, \omega)$ defined as

$$
\mathcal{A}=\left(\begin{array}{ll}
\mathcal{A}_{11} & \mathcal{A}_{12} \\
\mathcal{A}_{21} & \mathcal{A}_{22}
\end{array}\right) .
$$

To this end, we separate the derivatives in the $x_{3}$-direction from the derivatives in the $x_{1^{-}}$ direction in equations (B1) and (B2), the latter multiplied by $\vartheta_{33}^{-1} \vartheta_{3 s}$ on both sides. Hence,

$$
\begin{aligned}
& \partial_{3} Q_{3}=i \omega \alpha P+i \omega \gamma_{r} Q_{r}-\partial_{1} Q_{1}+B \\
& \partial_{3} P=-\vartheta_{33}^{-1}\left(-i \omega Q_{3}-i \omega \vartheta_{3 s} \gamma_{s} P+\vartheta_{31} \partial_{1} P-\vartheta_{3 s} C_{s}\right) .
\end{aligned}
$$


$Q_{1}$ needs to be eliminated from equation (B6). From equation (B2), multiplied on both sides by $\vartheta_{1 s}$, we obtain

$$
Q_{1}=\frac{1}{i \omega}\left(-i \omega \vartheta_{1 s} \gamma_{s} P+\vartheta_{1 s} \partial_{s} P-\vartheta_{1 s} C_{s}\right)
$$

Substitution of equation $(\mathrm{B} 8)$ into $(\mathrm{B} 6)$ gives

$$
\partial_{3} Q_{3}=i \omega \alpha P+i \omega \gamma_{3} Q_{3}-\frac{1}{i \omega}\left(\partial_{1}-i \omega \gamma_{1}\right)\left(-i \omega \vartheta_{1 s} \gamma_{s} P+\vartheta_{1 s} \partial_{s} P-\vartheta_{1 s} C_{s}\right)+B
$$

or, upon substitution of equation (B7) and some reorganization,

$$
\begin{aligned}
\partial_{3} Q_{3} & =\left(i \omega \alpha-\frac{1}{i \omega}\left(\partial_{1}-i \omega \gamma_{1}\right) b_{1}\left(\partial_{1}-i \omega \gamma_{1}\right)\right) P \\
& +\left(i \omega \gamma_{3}-\left(\partial_{1}-i \omega \gamma_{1}\right) \vartheta_{13} \vartheta_{33}^{-1}\right) Q_{3}+B+\frac{1}{i \omega}\left(\partial_{1}-i \omega \gamma_{1}\right) b_{s} C_{s},
\end{aligned}
$$

with

$$
b_{s}=\vartheta_{1 s}-\vartheta_{13} \vartheta_{33}^{-1} \vartheta_{3 s}
$$

or, using equations A74 - A77),

$$
\begin{aligned}
& b_{1}=1 / \beta_{11}, \\
& b_{3}=0 .
\end{aligned}
$$

Equations (B7) and (B10) can be cast in the form of the matrix-vector wave equation defined in equations $(\mathrm{B} 3)-(\mathrm{B} 5)$, with

$$
\begin{aligned}
\mathcal{A}_{11} & =i \omega \gamma_{3}-d\left(\partial_{1}-i \omega \gamma_{1}\right) \\
\mathcal{A}_{12} & =i \omega \vartheta_{33}^{-1} \\
\mathcal{A}_{21} & =i \omega \alpha-\frac{1}{i \omega}\left(\partial_{1}-i \omega \gamma_{1}\right) b_{1}\left(\partial_{1}-i \omega \gamma_{1}\right), \\
\mathcal{A}_{22} & =i \omega \gamma_{3}-\left(\partial_{1}-i \omega \gamma_{1}\right) d \\
C^{o} & =d C_{1}+C_{3} \\
B^{o} & =B+\frac{1}{i \omega}\left(\partial_{1}-i \omega \gamma_{1}\right) b_{1} C_{1}
\end{aligned}
$$

with

$$
d=\vartheta_{33}^{-1} \vartheta_{13}=-\beta_{31} / \beta_{11}
$$


The notation in the right-hand side of equations $(\mathrm{B} 14)-(\mathrm{B} 17)$ should be understood in the sense that differential operators act on all factors to the right of it. For example, the operator $\partial_{1} b_{1} \partial_{1}$ in equation $(\overline{\mathrm{B} 16})$, applied via equation $(\mathrm{B} 3)$ to the wave field $P$, implies $\partial_{1}\left(b_{1} \partial_{1} P\right)$.

\section{Decomposition of the operator matrix}

We use equation (45) to transform the operator matrix $\mathcal{A}$ defined in equation (B5) to the slowness domain, assuming the medium is laterally invariant at depth level $x_{3}$. The spatial differential operators $\partial_{1}$ are thus replaced by $i \omega s_{1}$, hence

$$
\tilde{\mathcal{A}}\left(s_{1}, x_{3}, \omega\right)=\left(\begin{array}{cc}
i \omega\left\{\gamma_{3}-d\left(s_{1}-\gamma_{1}\right)\right\} & i \omega \vartheta_{33}^{-1} \\
i \omega \vartheta_{33} s_{3}^{2} & i \omega\left\{\gamma_{3}-d\left(s_{1}-\gamma_{1}\right)\right\}
\end{array}\right)
$$

with

$$
s_{3}^{2}=\vartheta_{33}^{-1}\left(\alpha-b_{1}\left(s_{1}-\gamma_{1}\right)^{2}\right)
$$

The eigenvalue decomposition of $\tilde{\mathcal{A}}$ reads

$$
\tilde{\mathcal{A}}=\tilde{\mathcal{L}} \tilde{\mathcal{H}} \tilde{\mathcal{L}}^{-1}
$$

Using the standard approach to find eigenvalues and eigenvectors we obtain

$$
\begin{aligned}
\tilde{\mathcal{H}}\left(s_{1}, x_{3}, \omega\right) & =\left(\begin{array}{cc}
i \omega \lambda^{+} & 0 \\
0 & -i \omega \lambda^{-}
\end{array}\right), \\
\tilde{\mathcal{L}}\left(s_{1}, x_{3}, \omega\right) & =\frac{1}{\sqrt{2}}\left(\begin{array}{cc}
1 / \sqrt{\vartheta_{33} s_{3}} & 1 / \sqrt{\vartheta_{33} s_{3}} \\
\sqrt{\vartheta_{33} s_{3}} & -\sqrt{\vartheta_{33} s_{3}}
\end{array}\right), \\
\left\{\tilde{\mathcal{L}}\left(s_{1}, x_{3}, \omega\right)\right\}^{-1} & =\frac{1}{\sqrt{2}}\left(\begin{array}{cc}
\sqrt{\vartheta_{33} s_{3}} & 1 / \sqrt{\vartheta_{33} s_{3}} \\
\sqrt{\vartheta_{33} s_{3}} & -1 / \sqrt{\vartheta_{33} s_{3}}
\end{array}\right),
\end{aligned}
$$

where

$$
\begin{aligned}
\lambda^{ \pm} & =s_{3} \pm\left\{\gamma_{3}-d\left(s_{1}-\gamma_{1}\right)\right\}, \\
s_{3} & = \begin{cases}\sqrt{\vartheta_{33}^{-1}\left(\alpha-b_{1}\left(s_{1}-\gamma_{1}\right)^{2}\right)}, & \text { for } \quad\left(s_{1}-\gamma_{1}\right)^{2} \leq \frac{\alpha}{b_{1}}, \\
i \sqrt{\vartheta_{33}^{-1}\left(b_{1}\left(s_{1}-\gamma_{1}\right)^{2}-\alpha\right)}, & \text { for } \quad\left(s_{1}-\gamma_{1}\right)^{2}>\frac{\alpha}{b_{1}}\end{cases}
\end{aligned}
$$


Note that the intervals $\left(s_{1}-\gamma_{1}\right)^{2} \leq \frac{\alpha}{b_{1}}$ and $\left(s_{1}-\gamma_{1}\right)^{2}>\frac{\alpha}{b_{1}}$ in equation B28 correspond to propagating and evanescent waves, respectively.

\section{Reciprocity theorems for decomposed wave fields}

We derive reciprocity theorems for downgoing and upgoing flux-normalized wave fields,

exploiting the symmetry properties of operator $\tilde{\mathcal{L}}$. Reciprocity theorems $(17)$ and $(18)$ can be compactly written as

$$
\int_{\partial \mathbb{D}_{0}}\left\{\mathbf{q}_{A}^{(\mathrm{c})}\right\}^{t} \mathbf{N} \mathbf{q}_{B} \mathrm{~d} \mathbf{x}=\int_{\partial \mathbb{D}_{A}}\left\{\mathbf{q}_{A}^{(\mathrm{c})}\right\}^{t} \mathbf{N} \mathbf{q}_{B} \mathrm{~d} \mathbf{x}
$$

and

$$
\int_{\partial \mathbb{D}_{0}} \mathbf{q}_{A}^{\dagger} \mathbf{K} \mathbf{q}_{B} \mathrm{~d} \mathbf{x}=\int_{\partial \mathbb{D}_{A}} \mathbf{q}_{A}^{\dagger} \mathbf{K} \mathbf{q}_{B} \mathrm{~d} \mathbf{x}
$$

with $\mathbf{q}$ defined in equation (20), superscript $t$ denoting transposition, $\dagger$ transposition and complex conjugation, and matrices $\mathbf{N}$ and $\mathbf{K}$ defined as

$$
\mathbf{N}=\left(\begin{array}{cc}
0 & 1 \\
-1 & 0
\end{array}\right), \quad \mathbf{K}=\left(\begin{array}{ll}
0 & 1 \\
1 & 0
\end{array}\right)
$$

According to equation (19), vector $\mathbf{q}$ is (for both states) related to vector $\mathbf{p}$ via $\mathbf{q}=\mathcal{L} \mathbf{p}$, with $\mathbf{p}$ defined in equation (20). Here we use this relation and the symmetry properties of composition operator $\tilde{\mathcal{L}}$ to recast equations B29 and B30 into reciprocity theorems for downgoing and upgoing wave fields.

Using the spatial Fourier transform, defined in equation (45), and Parseval's theorem, we first rewrite the integrals in equations $(\mathrm{B} 29)$ and $(\mathrm{B} 30)$ as

$$
\begin{aligned}
\int_{-\infty}^{\infty}\left\{\mathbf{q}_{A}^{(\mathrm{c})}\left(x_{1}, x_{3}, \omega\right)\right\}^{t} \mathbf{N} \mathbf{q}_{B}\left(x_{1}, x_{3}, \omega\right) \mathrm{d} x_{1}= \\
\\
\quad \frac{\omega}{2 \pi} \int_{-\infty}^{\infty}\left\{\tilde{\mathbf{q}}_{A}^{(\mathrm{c})}\left(-s_{1}, x_{3}, \omega\right)\right\}^{t} \mathbf{N} \tilde{\mathbf{q}}_{B}\left(s_{1}, x_{3}, \omega\right) \mathrm{d} s_{1}
\end{aligned}
$$


and

$$
\begin{aligned}
\int_{-\infty}^{\infty}\left\{\mathbf{q}_{A}\left(x_{1}, x_{3}, \omega\right)\right\}^{\dagger} \mathbf{K} \mathbf{q}_{B}\left(x_{1}, x_{3}, \omega\right) \mathrm{d} x_{1}= \\
\\
\quad \frac{\omega}{2 \pi} \int_{-\infty}^{\infty}\left\{\tilde{\mathbf{q}}_{A}\left(s_{1}, x_{3}, \omega\right)\right\}^{\dagger} \mathbf{K} \tilde{\mathbf{q}}_{B}\left(s_{1}, x_{3}, \omega\right) \mathrm{d} s_{1}
\end{aligned}
$$

respectively, where $x_{3}$ can represent the depth level of $\partial \mathbb{D}_{0}$ or $\partial \mathbb{D}_{A}$. Assuming the medium parameters are laterally invariant at $x_{3}$, the composition operation $\mathbf{q}=\mathcal{L} \mathbf{p}$ can be rewritten in the slowness domain as

$$
\tilde{\mathbf{q}}\left(s_{1}, x_{3}, \omega\right)=\tilde{\mathcal{L}}\left(s_{1}, x_{3}, \omega\right) \tilde{\mathbf{p}}\left(s_{1}, x_{3}, \omega\right)
$$

with $\tilde{\mathcal{L}}\left(s_{1}, x_{3}, \omega\right)$ defined in equation $\left.\mathrm{B} 25\right)$. Substituting this in the right-hand sides of equations (B32) and (B33) yields

$$
\begin{aligned}
& \frac{\omega}{2 \pi} \int_{-\infty}^{\infty}\left\{\tilde{\mathbf{q}}_{A}^{(\mathrm{c})}\left(-s_{1}, x_{3}, \omega\right)\right\}^{t} \mathbf{N} \tilde{\mathbf{q}}_{B}\left(s_{1}, x_{3}, \omega\right) \mathrm{d} s_{1}= \\
& \quad \frac{\omega}{2 \pi} \int_{-\infty}^{\infty}\left\{\tilde{\mathbf{p}}_{A}^{(\mathrm{c})}\left(-s_{1}, x_{3}, \omega\right)\right\}^{t}\left\{\tilde{\mathcal{L}}^{(\mathrm{c})}\left(-s_{1}, x_{3}, \omega\right)\right\}^{t} \mathbf{N} \tilde{\mathcal{L}}\left(s_{1}, x_{3}, \omega\right) \tilde{\mathbf{p}}_{B}\left(s_{1}, x_{3}, \omega\right) \mathrm{d} s_{1}
\end{aligned}
$$

and

$$
\begin{aligned}
& \frac{\omega}{2 \pi} \int_{-\infty}^{\infty}\left\{\tilde{\mathbf{q}}_{A}\left(s_{1}, x_{3}, \omega\right)\right\}^{\dagger} \mathbf{K} \tilde{\mathbf{q}}_{B}\left(s_{1}, x_{3}, \omega\right) \mathrm{d} s_{1}= \\
& \quad \frac{\omega}{2 \pi} \int_{-\infty}^{\infty}\left\{\tilde{\mathbf{p}}_{A}\left(s_{1}, x_{3}, \omega\right)\right\}^{\dagger}\left\{\tilde{\mathcal{L}}\left(s_{1}, x_{3}, \omega\right)\right\}^{\dagger} \mathbf{K} \tilde{\mathcal{L}}\left(s_{1}, x_{3}, \omega\right) \tilde{\mathbf{p}}_{B}\left(s_{1}, x_{3}, \omega\right) \mathrm{d} s_{1},
\end{aligned}
$$

respectively. From the definition of $\tilde{\mathcal{L}}\left(s_{1}, x_{3}, \omega\right)$ in equation $\mathrm{B} 25$, with $s_{3}$ defined in equation (B28), recalling that superscript (c) implies that $\gamma_{r}$ is replaced by $-\gamma_{r}$, we find

$$
\begin{gathered}
\left\{\tilde{\mathcal{L}}^{(\mathrm{c})}\left(-s_{1}, x_{3}, \omega\right)\right\}^{t} \mathbf{N} \tilde{\mathcal{L}}\left(s_{1}, x_{3}, \omega\right)=-\mathbf{N}, \quad \text { for } \quad-\infty<s_{1}<\infty \\
\left\{\tilde{\mathcal{L}}\left(s_{1}, x_{3}, \omega\right)\right\}^{\dagger} \mathbf{K} \tilde{\mathcal{L}}\left(s_{1}, x_{3}, \omega\right)=\mathbf{J}, \quad \text { for } \quad\left(s_{1}-\gamma_{1}\right)^{2} \leq \frac{\alpha}{b_{1}}
\end{gathered}
$$

with $\mathbf{J}$ defined as

$$
\mathbf{J}=\left(\begin{array}{cc}
1 & 0 \\
0 & -1
\end{array}\right)
$$

Note that equation (B37) holds for propagating and evanescent waves, whereas equation (B38) holds for propagating waves only. Substituting equations (B37) and (B38) into equa- 
tions (B35) and (B36) and using Parseval's theorem again yields

$$
\begin{aligned}
\int_{-\infty}^{\infty}\left\{\mathbf{q}_{A}^{(\mathrm{c})}\left(x_{1}, x_{3}, \omega\right)\right\}^{t} \mathbf{N} \mathbf{q}_{B}\left(x_{1}, x_{3}, \omega\right) \mathrm{d} x_{1}= \\
\quad-\int_{-\infty}^{\infty}\left\{\mathbf{p}_{A}^{(\mathrm{c})}\left(x_{1}, x_{3}, \omega\right)\right\}^{t} \mathbf{N p}_{B}\left(x_{1}, x_{3}, \omega\right) \mathrm{d} x_{1}
\end{aligned}
$$

and

$$
\begin{aligned}
\int_{-\infty}^{\infty}\left\{\mathbf{q}_{A}\left(x_{1}, x_{3}, \omega\right)\right\}^{\dagger} \mathbf{K} \mathbf{q}_{B}\left(x_{1}, x_{3}, \omega\right) \mathrm{d} x_{1}= \\
\int_{-\infty}^{\infty}\left\{\mathbf{p}_{A}\left(x_{1}, x_{3}, \omega\right)\right\}^{\dagger} \mathbf{J} \mathbf{p}_{B}\left(x_{1}, x_{3}, \omega\right) \mathrm{d} x_{1},
\end{aligned}
$$

respectively. Equation (B40) is exact, whereas in equation (B41) evanescent waves are neglected. Using these equations at boundaries $\partial \mathbb{D}_{0}$ and $\partial \mathbb{D}_{A}$ in reciprocity theorems $(\mathrm{B} 29)$ and (B30) yields

$$
\int_{\partial \mathbb{D}_{0}}\left\{\mathbf{p}_{A}^{(\mathrm{c})}\right\}^{t} \mathbf{N} \mathbf{p}_{B} \mathrm{~d} \mathbf{x}=\int_{\partial \mathbb{D}_{A}}\left\{\mathbf{p}_{A}^{(\mathrm{c})}\right\}^{t} \mathbf{N} \mathbf{p}_{B} \mathrm{~d} \mathbf{x}
$$

and

$$
\int_{\partial \mathbb{D}_{0}} \mathbf{p}_{A}^{\dagger} \mathbf{J} \mathbf{p}_{B} \mathrm{~d} \mathbf{x}=\int_{\partial \mathbb{D}_{A}} \mathbf{p}_{A}^{\dagger} \mathbf{J} \mathbf{p}_{B} \mathrm{~d} \mathbf{x}
$$

respectively. Substituting the expressions for $\mathbf{p}$ (equation 20), $\mathbf{N}$ (equation B31) and $\mathbf{J}$ (equation B39) we obtain the reciprocity theorems of equations (21) and (22) for the downgoing and upgoing fields $U^{+}$and $U^{-}$. 


\section{References}

[1] J. F. Claerbout, "Toward a unified theory of reflector mapping", Geophysics 36, 467-481 (1971).

[2] R. H. Stolt, "Migration by Fourier transform", Geophysics 43, 23-48 (1978).

[3] A. J. Berkhout and D. W. van Wulfften Palthe, "Migration in terms of spatial deconvolution", Geophys. Prosp. 27, 261-291 (1979).

[4] E. G. Williams and J. D. Maynard, "Holographic imaging without the wavelength resolution limit", Phys. Rev. Lett. 45, 554-557 (1980).

[5] A. J. Devaney, "A filtered backpropagation algorithm for diffraction tomography", Ultrasonic Imaging 4, 336-350 (1982).

[6] N. Bleistein and J. K. Cohen, "Velocity inversion - Present status, new directions", Geophysics 47, 1497-1511 (1982).

[7] J. D. Maynard, E. G. Williams, and Y. Lee, "Nearfield acoustic holography: I. Theory of generalized holography and the development of NAH", J. Acoust. Soc. Am. 78, 1395-1413 (1985).

[8] K. J. Langenberg, M. Berger, T. Kreutter, K. Mayer, and V. Schmitz, "Synthetic aperture focusing technique signal processing", NDT International 19, 177-189 (1986).

[9] G. A. McMechan, "Migration by extrapolation of time-dependent boundary values", Geophys. Prosp. 31, 413-420 (1983).

[10] C. Esmersoy and M. Oristaglio, "Reverse-time wave-field extrapolation, imaging, and inversion", Geophysics 53, 920-931 (1988).

[11] M. L. Oristaglio, "An inverse scattering formula that uses all the data", Inverse Probl. 5, 1097-1105 (1989).

[12] S. J. Norton, "Annular array imaging with full-aperture resolution", J. Acoust. Soc. Am. 92, 3202-3206 (1992).

[13] S. F. Wu, "Hybrid near-field acoustic holography", J. Acoust. Soc. Am. 115, 207-217 (2004).

[14] C. Lindsey and D. C. Braun, "Principles of seismic holography for diagnostics of the shallow subphotosphere", Astrophys. J. Suppl. Series 155, 209-225 (2004).

[15] J. Etgen, S. H. Gray, and Y. Zhang, "An overview of depth imaging in exploration geophysics", Geophysics 74, WCA5-WCA17 (2009).

[16] D. J. Verschuur, A. J. Berkhout, and C. P. A. Wapenaar, "Adaptive surface-related multiple elimination", Geophysics 57, 1166-1177 (1992).

[17] P. M. Carvalho, A. B. Weglein, and R. H. Stolt, "Nonlinear inverse scattering for multiple 
suppression: Application to real data, Part 1", in SEG, Expanded Abstracts, 1093-1095 (1992).

[18] R. G. van Borselen, J. T. Fokkema, and P. M. van den Berg, "Removal of surface-related wave phenomena - The marine case", Geophysics 61, 202-210 (1996).

[19] J. Biersteker, "MAGIC: Shell's surface multiple attenuation technique", in SEG, Expanded Abstracts, 1301-1304 (2001).

[20] A. Pica, G. Poulain, B. David, M. Magesan, S. Baldock, T. Weisser, P. Hugonnet, and P. Herrmann, "3D surface-related multiple modeling", The Leading Edge 24, 292-296 (2005).

[21] B. Dragoset, E. Verschuur, I. Moore, and R. Bisley, "A perspective on 3D surface-related multiple elimination", Geophysics 75, 75A245-75A261 (2010).

[22] A. B. Weglein, F. A. Gasparotto, P. M. Carvalho, and R. H. Stolt, "An inverse-scattering series method for attenuating multiples in seismic reflection data", Geophysics 62, 1975-1989 (1997).

[23] F. Ten Kroode, "Prediction of internal multiples", Wave Motion 35, 315-338 (2002).

[24] A. B. Weglein, F. V. Araújo, P. M. Carvalho, R. H. Stolt, K. H. Matson, R. T. Coates, D. Corrigan, D. J. Foster, S. A. Shaw, and H. Zhang, "Inverse scattering series and seismic exploration", Inverse Probl. 19, R27-R83 (2003).

[25] A. J. Berkhout, "Review paper: An outlook on the future of seismic imaging, Part II: FullWavefield Migration", Geophys. Prosp. 62, 931-949 (2014).

[26] M. Davydenko and D. J. Verschuur, "Full-wavefield migration: using surface and internal multiples in imaging", Geophys. Prosp. 65, 7-21 (2017).

[27] J. H. Rose, " "Single-sided" focusing of the time-dependent Schrödinger equation", Phys. Rev. A 65, 012707 (2001).

[28] J. H. Rose, "'Single-sided' autofocusing of sound in layered materials", Inverse Probl. 18, 1923-1934 (2002).

[29] F. Broggini and R. Snieder, "Connection of scattering principles: a visual and mathematical tour", European Journal of Physics 33, 593-613 (2012).

[30] K. Wapenaar, F. Broggini, and R. Snieder, "Creating a virtual source inside a medium from reflection data: heuristic derivation and stationary-phase analysis", Geophys. J. Int. 190, 1020-1024 (2012).

[31] K. Wapenaar, J. Thorbecke, J. van der Neut, F. Broggini, E. Slob, and R. Snieder, "Green's function retrieval from reflection data, in absence of a receiver at the virtual source position", J. Acoust. Soc. Am. 135, 2847-2861 (2014).

[32] F. Broggini, R. Snieder, and K. Wapenaar, "Data-driven wavefield focusing and imaging with multidimensional deconvolution: Numerical examples for reflection data with internal multiples", Geophysics 79, WA107-WA115 (2014). 
[33] J. Behura, K. Wapenaar, and R. Snieder, "Autofocus imaging: Image reconstruction based on inverse scattering theory", Geophysics 79, A19-A26 (2014).

[34] G. A. Meles, K. Löer, M. Ravasi, A. Curtis, and C. A. da Costa Filho, "Internal multiple prediction and removal using Marchenko autofocusing and seismic interferometry", Geophysics 80, A7-A11 (2015).

[35] J. van der Neut, I. Vasconcelos, and K. Wapenaar, "On Green's function retrieval by iterative substitution of the coupled Marchenko equations", Geophys. J. Int. 203, 792-813 (2015).

[36] J. van der Neut and K. Wapenaar, "Adaptive overburden elimination with the multidimensional Marchenko equation", Geophysis 81, T265-T284 (2016).

[37] J. Thorbecke, E. Slob, J. Brackenhoff, J. van der Neut, and K. Wapenaar, "Implementation of the Marchenko method", Geophysics 82, WB29-WB45 (2017).

[38] J. Van der Neut, M. Ravasi, Y. Liu, and I. Vasconcelos, "Target-enclosed seismic imaging", Geophysics 82, Q53-Q66 (2017).

[39] S. Singh, R. Snieder, J. van der Neut, J. Thorbecke, E. Slob, and K. Wapenaar, "Accounting for free-surface multiples in Marchenko imaging", Geophysics 82, R19-R30 (2017).

[40] C. Mildner, F. Broggini, J. O. A. Robertsson, D. J. van Manen, and S. Greenhalgh, "Targetoriented velocity analysis using Marchenko-redatumed data", Geophysics 82, R75-R86 (2017).

[41] P. Elison, D. J. van Manen, J. O. A. Robertsson, M. S. Dukalski, and K. de Vos, "Marchenkobased immersive wave simulation", Geophys. J. Int. 215, 1118-1131 (2018).

[42] M. Ravasi, I. Vasconcelos, A. Kritski, A. Curtis, C. A. da Costa Filho, and G. A. Meles, "Target-oriented Marchenko imaging of a North Sea field", Geophys. J. Int. 205, 99-104 (2016).

[43] M. Ravasi, "Rayleigh-Marchenko redatuming for target-oriented, true-amplitude imaging", Geophysics 82, S439-S452 (2017).

[44] M. Staring, R. Pereira, H. Douma, J. van der Neut, and K. Wapenaar, "Source-receiver Marchenko redatuming on field data using an adaptive double-focusing method", Geophysics 83, S579-S590 (2018).

[45] J. Brackenhoff, J. Thorbecke, and K. Wapenaar, "Monitoring induced distributed doublecouple sources using Marchenko-based virtual receivers", Solid Earth 10, doi.org/10.5194/se2018-142 (2019).

[46] K. Wapenaar, J. Brackenhoff, J. Thorbecke, J. van der Neut, E. Slob, and E. Verschuur, "Virtual acoustics in inhomogeneous media with single-sided access", Scientific Reports 8, 2497 (2018).

[47] T. Cui, T. S. Becker, D.-J. van Manen, J. E. Rickett, and I. Vasconcelos, "Marchenko redatuming in a dissipative medium: Numerical and experimental implementation", Phys. Rev. 
Appl. 10, 044022 (2018).

[48] J. R. Willis, "Effective constitutive relations for waves in composites and metamaterials", Proc. R. Soc. A 467, 1865-1879 (2011).

[49] C. He, M. H. Lu, X. Heng, L. Feng, and Y. F. Chen, "Parity-time electromagnetic diodes in a two-dimensional nonreciprocal photonic crystal”, Phys. Rev. B 83, 075117 (2011).

[50] A. G. Ardakani, "Nonreciprocal electromagnetic wave propagation in one-dimensional ternary magnetized plasma photonic crystals", J. Opt. Soc. Am. B 31, 332-339 (2014).

[51] J. R. Willis, "The construction of effective relations for waves in a composite", Comptes Rendus Mecanique 340, 181-192 (2012).

[52] A. N. Norris, A. L. Shuvalov, and A. A. Kutsenko, "Analytical formulation of threedimensional dynamic homogenization for periodic elastic systems", Proc. R. Soc. A 468, 1629-1651 (2012).

[53] Z. Gu, J. Hu, B. Liang, X. Zou, and J. Cheng, "Broadband non-reciprocal transmission of sound with invariant frequency", Scientific Reports 6, 19824 (2016).

[54] G. Trainiti and M. Ruzzene, "Non-reciprocal elastic wave propagation in spatiotemporal periodic structures", New Journal of Physics 18, 083047 (2016).

[55] H. Nassar, H. Chen, A. N. Norris, M. R. Haberman, and G. L. Huang, "Non-reciprocal wave propagation in modulated elastic metamaterials", Proc. R. Soc. A 473, 20170188 (2017).

[56] H. Nassar, X. C. Xu, A. N. Norris, and G. L. Huang, "Modulated phononic crystals: Nonreciprocal wave propagation and Willis materials", J. Mech. Phys. Solids 101, 10-29 (2017).

[57] M. A. Attarzadeh and M. Nouh, "Non-reciprocal elastic wave propagation in 2D phononic membranes with spatiotemporally varying material properties", J. Sound Vibr. 422, 264-277 (2018).

[58] J. T. Fokkema and P. M. van den Berg, Seismic applications of acoustic reciprocity (Elsevier, Amsterdam) (1993), Chap. 5.

[59] A. T. de Hoop, Handbook of radiation and scattering of waves (Academic Press, London) (1995), Chaps. 7, 15 and 28.

[60] J. D. Achenbach, Reciprocity in elastodynamics (Cambridge University Press) (2003), Chaps. 4 and 6.

[61] J. A. Kong, "Theorems of bianisotropic media", Proc. IEEE 60, 1036-1046 (1972).

[62] R. R. Birss and R. G. Shrubsall, "The propagation of EM waves in magnetoelectric crystals", Phil. Mag. 15, 687-700 (1967).

[63] I. V. Lindell, A. H. Sihvola, and K. Suchy, "Six-vector formalism in electromagnetics of bianisotropic media", J. Electr. Waves and Appl. 9, 887-903 (1995).

[64] C. Altman and K. Suchy, Reciprocity, spatial mapping and time reversal in electromagnetics 
(Kluwer, Dordrecht) (1991), Chap. 3.

[65] E. Slob and K. Wapenaar, "Retrieving the Green's function from cross correlation in a bianisotropic medium", Progress In Electromagnetics Research, PIER 93, 255-274 (2009).

[66] L. M. Lyamshev, "On some integral relationships in acoustics of moving medium", Dokl. Ak. Nauk 138, 575-578 (1961).

[67] O. A. Godin, "Reciprocity and energy theorems for waves in a compressible inhomogeneous moving fluid", Wave Motion 25, 143-167 (1997).

[68] K. Wapenaar and J. Fokkema, "Reciprocity theorems for diffusion, flow and waves", J. Appl. Mech. 71, 145-150 (2004).

[69] C. P. A. Wapenaar and A. J. Berkhout, Elastic wave field extrapolation (Elsevier, Amsterdam) (1989), Chap. 3 and App. B.

[70] J. P. Corones, M. E. Davison, and R. J. Krueger, "Direct and inverse scattering in the time domain via invariant imbedding equations", J. Acoust. Soc. Am. 74, 1535-1541 (1983).

[71] L. Fishman, J. J. McCoy, and S. C. Wales, "Factorization and path integration of the Helmholtz equation: Numerical algorithms", J. Acoust. Soc. Am. 81, 1355-1376 (1987).

[72] L. Fishman, "One-way propagation methods in direct and inverse scalar wave propagation modeling", Radio Science 28, 865-876 (1993).

[73] M. V. de Hoop, Directional decomposition of transient acoustic wave fields, Ph.D. thesis, Delft University of Technology (repository.tudelft.nl) (1992).

[74] M. V. de Hoop, "Generalization of the Bremmer coupling series", J. Math. Phys. 37, 32463282 (1996).

[75] C. P. A. Wapenaar, "Reciprocity theorems for two-way and one-way wave vectors: a comparison", J. Acoust. Soc. Am. 100, 3508-3518 (1996).

[76] A. J. Haines and M. V. de Hoop, "An invariant imbedding analysis of general wave scattering problems", J. Math. Phys. 37, 3854-3881 (1996).

[77] L. Fishman, M. V. de Hoop, and M. J. N. van Stralen, "Exact constructions of square-root Helmholtz operator symbols: The focusing quadratic profile”, J. Math. Phys. 41, 4881-4938 (2000).

[78] E. Slob, K. Wapenaar, F. Broggini, and R. Snieder, "Seismic reflector imaging using internal multiples with Marchenko-type equations", Geophysics 79, S63-S76 (2014).

[79] K. Wapenaar, J. Fokkema, M. Dillen, and P. Scherpenhuijsen, "One-way acoustic reciprocity and its applications in multiple elimination and time-lapse seismics", in $S E G$, Expanded $A b$ stracts, 2377-2380 (2000).

[80] L. Amundsen, "Elimination of free-surface related multiples without need of the source wavelet", Geophysics 66, 327-341 (2001). 
[81] E. Holvik and L. Amundsen, "Elimination of the overburden response from multicomponent source and receiver seismic data, with source designature and decomposition into PP-, PS-, SP-, and SS-wave responses", Geophysics 70, S43-S59 (2005).

[82] K. Wapenaar and J. van der Neut, "A representation for Green's function retrieval by multidimensional deconvolution", J. Acoust. Soc. Am. 128, EL366-EL371 (2010).

[83] J. van der Neut, J. Thorbecke, K. Mehta, E. Slob, and K. Wapenaar, "Controlled-source interferometric redatuming by crosscorrelation and multidimensional deconvolution in elastic media", Geophysics 76, SA63-SA76 (2011).

[84] M. Ravasi, G. Meles, A. Curtis, Z. Rawlinson, and L. Yikuo, "Seismic interferometry by multidimensional deconvolution without wavefield separation", Geophys. J. Int. 202, 1-16 (2015).

[85] B. L. N. Kennett and N. J. Kerry, "Seismic waves in a stratified half-space", Geophys. J. R. Astr. Soc. 57, 557-584 (1979).

[86] R. M. Kiehn, G. P. Kiehn, and J. B. Roberds, "Parity and time-reversal symmetry breaking, singular solutions, and Fresnel surfaces", Phys. Rev. A 43, 5665-5671 (1991).

[87] E. Slob and K. Wapenaar, "Green's function extraction for interfaces with impedance boundary conditions", IEEE Trans. Ant. Prop. 60, 351-359 (2012).

[88] B. D. H. Tellegen, "The gyrator, a new electric network element", Philips Res. Rep. 3, 81-101 (1948). 